ANL-HEP-PR-02-115

EFI- $02-43$

FERMILAB-PUB-02/342-T

\title{
Opaque Branes in Warped Backgrounds
}

\author{
Marcela Carena ${ }^{a}$, Eduardo Pontón $^{a}$, \\ Tim M.P. Tait ${ }^{a}$, and C.E.M. Wagner ${ }^{b, c}$ \\ ${ }^{a}$ Fermi National Accelerator Laboratory, P.O. Box 500, Batavia, IL 60510, USA \\ ${ }^{b}$ HEP Division, Argonne National Laboratory, 9700 Cass Ave., Argonne, IL 60439, USA \\ ${ }^{c}$ Enrico Fermi Institute, Univ. of Chicago, 5640 Ellis Ave., Chicago, IL 60637, USA
}

September 26, 2018

\begin{abstract}
We examine localized kinetic terms for gauge fields which can propagate into compact, warped extra dimensions. We show that these terms can have a relevant impact on the values of the Kaluza-Klein (KK) gauge field masses, wave functions, and couplings to brane and bulk matter. The resulting phenomenological implications are discussed. In particular, we show that the presence of opaque branes, with non-vanishing brane-localized gauge kinetic terms, allow much lower values of the lightest KK mode than in the case of transparent branes. Moreover, we show that if the large discrepancies among the different determinations of the weak mixing angle would be solved in favor of the value obtained from the lepton asymmetries, bulk electroweak gauge fields in warped-extra dimensions may lead to an improvement of the agreement of the fit to the electroweak precision data for a Higgs mass of the order of the weak scale and a mass of the first KK gauge boson excitation of a few TeV, most likely within reach of the LHC.
\end{abstract}




\section{Introduction}

Much of the recent interest in theories with extra dimensions stems from the fact that they provide a possible solution to the hierarchy problem of the Standard Model. In the case of flat extra dimensions [1, the fundamental Planck scale could take values of order of the weak scale. The gravity interactions at long distances are governed by the four dimensional Planck scale, $M_{P l}$, which is related to the fundamental Planck scale $M$ by a factor proportional to the volume of the extra dimensional space. Therefore, for large values of the compactification scale and/or large number of extra dimensions, one can reconcile the observed value of the Planck scale with a fundamental scale $M \simeq \mathcal{O}(\mathrm{TeV})$.

Since this mechanism demands an a priori unexplained large extra-dimensional volume, it could be argued that rather than providing a solution to the hierarchy problem, theories with flat extra dimensions allow a reformulation of the problem. On the other hand, flat extra dimensions would only be possible in the presence of a tension-less brane, confining all SM fields. Under these conditions, however, it is somewhat more natural to think about a brane with finite tension, and an induced curvature in the extra dimensional scenarios.

Non-vanishing curvature provides a distortion of the metric, which allows new alternatives to address the hierarchy problem. For instance, one can find simple solutions to the case of branes with finite tension by assuming that the local four dimensional metric is affected by an exponential warp factor, which depends linearly on the position of the brane. In this simple famework, one can get a solution to the hierarchy problem without assuming any unnatural large factor [2]. Indeed, assuming all fundamental mass scales to be of the same order, the ratio of the physical Higgs vacuum expectation value to the observable four dimensional Planck scale is exponentially suppressed, with an exponent that depends on the warp factor $k$ times the position $L$ of the Higgs brane in the extra dimensions. Taking $k L \simeq 34.5$ provides a good solution to the hierarchy problem. It is important to emphasize that, as in any other solution, new physics appears at the $\mathrm{TeV}$ scale. This new physics includes graviton Kaluza Klein states and a graviscalar, also called the radion, with effective interactions suppressed by a scale of order of the $\mathrm{TeV}, M e^{-k L}$.

It is natural to assume that not only the Higgs field, but all matter fields are confined to the brane at $y=L$, which we shall call the infrared brane. The gauge fields, however, may propagate in the extra dimensions. One motivation for this is the fact that, from the field theoretical point of view, it is more difficult to localize gauge fields than to localize fermions. 
However, there is a phenomenological obstacle to this realization : the Kaluza Klein excitations of the gauge bosons couple with a strength $\sqrt{2 k L}$ times the zero mode coupling, an order of magnitude larger for values of $k L$ necessary to provide a solution of the hierarchy problem. The exchange of these strongly coupled higher KK states can be used to set a lower bound on the mass of the first KK excitation that is about $20 \mathrm{TeV}$ [3].

Precision electroweak observables present additional challenges, since these observables are affected at the tree-level [4, 5, 6]. The modification of these observables is related to the shape of the extra dimensional wave functions in the presence of a localized symmety breaking VEV. The presence of the localized scalar VEV induces a repulsion of the gauge fields from the brane, leading to a breakdown of the usual linear relation between the gauge boson mass with its coupling and the Higgs VEV. In order to recover acceptable phenomenological predictions, the bound on the first KK excitation has to be pushed to even larger values than the ones obtained from the KK boson exchange.

In this article we analyze how the results for the gauge field interactions described above is modified by the presence of local gauge field kinetic terms. Such terms are naturally expected to be present in any realistic theory, and indeed will be induced radiatively by the localized Higgs and fermions on the IR brane even if the underlying dynamics is such that they vanish at any particular scale [7, 8, 9]. The presence of a local brane kinetic term on the infrared brane implies that at sufficiently high energies, the gauge interactions on the brane should be four dimensional and renders the brane opaque to gauge fields of short wavelength along the extra dimension. This can only be possible if there is an important modification of the couplings of the gauge KK modes to matter localized on the brane [9, 10, 11]. Moreover, for sufficiently large local gauge kinetic terms, the physics on the brane should be dominated by the four dimensional behavior, and therefore it should be possible to relax these bounds on the mass of the lightest gauge boson KK mass.

The plan of this work is as follows. In section 2, we shall give the expression for the gauge field propagator with brane kinetic terms. We shall analyze its behavior in different momentum regimes and for different values of the local gauge kinetic terms. In section 3, we proceed with the Kaluza-Klein decomposition. After reviewing the situation in the case of transparent branes, in the absence of local gauge kinetic terms, we analyze the case of one or two opaque branes. We also provide a comparison of the results obtained with the KK decomposition with the ones derived from the behavior of the five dimensional gauge field propagator. In section 4 , we discuss the effects induced by the presence of a localized Higgs VEV. We discuss both the 
case of vanishing local gauge kinetic terms and the effects associated with the presence of these local terms. In section 5 we apply our results to the case where the electroweak gauge bosons propagate in the bulk, and analyze the phenomenological consequences in this scenario. In particular, we show that the presence of the brane-localized gauge kinetic terms opens the possibility for the first KK excitations to be within reach of the LHC. We reserve section 6 for the conclusions.

\section{Gauge Field Propagator with Brane Kinetic Terms}

In this section we derive the tree-level propagator for a gauge field described by the action

$$
S=-\frac{1}{4 g_{5}^{2}} \int d^{4} x d y \sqrt{-g}\left[\mathcal{F}^{M N} \mathcal{F}_{M N}+2 \delta(y) r_{U V} \mathcal{F}^{\mu \nu} \mathcal{F}_{\mu \nu}+2 \delta(y-L) r_{I R} \mathcal{F}^{\mu \nu} \mathcal{F}_{\mu \nu}\right]
$$

where $g$ is the determinant of the metric, and capital latin letters refer to the full $5 \mathrm{~d}$ coordinates, $M=0,1,2,3,5$, whereas lower case greek letters refer only to the four uncompactified dimensions, $\mu=0,1,2,3$. In the above we have rescaled the bulk kinetic term by absorbing $g_{5}$ into $\mathcal{A}_{M}$, so that $\mathcal{A}_{M}$ has mass dimension one, the canonical dimension for a gauge boson propagating in four dimensions. Then $g_{5}^{-2}$ has dimensions of mass and the coefficients of the local brane terms, $r_{i}=g_{5}^{2} / g_{i}^{2}$, have dimensions of length. ${ }^{1} \mathcal{F}$ is the usual field-strength functional of the gauge fields,

$$
\mathcal{F}_{M N}^{a}=\partial_{M} \mathcal{A}_{N}^{a}-\partial_{N} \mathcal{A}_{M}^{a}+f^{a b c} \mathcal{A}_{M}^{b} \mathcal{A}_{N}^{c}
$$

for a non-Abelian Yang-Mills theory, with the final term omitted in the Abelian case. For simplicity of notation, we denote the extra dimensional coordinate $x_{5}$ as $y$. The full set of five dimensional coordinates are denoted by capital letters, i.e. $X=\left\{x^{\mu}, y\right\}$.

We assume a background metric defined by the line element,

$$
d s^{2}=e^{-2 \sigma} \eta_{\mu \nu} d x^{\mu} d x^{\nu}+d y^{2}
$$

with $\eta_{\mu \nu}=\operatorname{diag}(-1,+1,+1,+1), \sigma(y)=k|y|$ and $0 \leq y \leq L$. The interval is assumed to arise from a $Z_{2}$ orbifold such that $\mathcal{A}_{\mu}$ is even and $\mathcal{A}_{5}$ is odd under reflection in $y$. It is then always possible to choose a gauge in which $\mathcal{A}_{5}=0$, corresponding to a unitary gauge. This will be

\footnotetext{
${ }^{1}$ For future convenience, we have factored out an explicit factor of two in the definition of the localized terms. The physical fifth dimension corresponds to the interval $[0, L]$, with the branes located at the endpoints. Consequently, each delta function contributes a factor of $1 / 2$ when performing the $y$ integration.
} 
sufficient for our purposes, but it is also interesting to exhibit the full gauge-dependence in an $R_{\xi}$ gauge.

In an $R_{\xi}$ gauge both the four dimensional vector field $\mathcal{A}_{\mu}$ and the scalar $\mathcal{A}_{5}$ propagate. The gauge choice is defined such that terms which mix the two are zero. This is enforced by the gauge-fixing term,

$$
-\frac{1}{2 \xi g_{5}^{2}}\left[\partial^{\mu} \mathcal{A}_{\mu}-\xi \partial_{y}\left(e^{-2 k y} \mathcal{A}_{5}\right)\right]^{2}
$$

We could work out the ghost interactions corresponding to this choice of gauge-fixing, but will not need them for our (tree-level) analyses. The propagators $G_{\mu \nu}\left(X, X^{\prime}\right)=\left\langle A_{\mu}(X) A_{\nu}\left(X^{\prime}\right)\right\rangle$ and $G_{55}\left(X, X^{\prime}\right)=\left\langle A_{5}(X) A_{5}\left(X^{\prime}\right)\right\rangle$, satisfy

$$
\begin{aligned}
\frac{1}{g_{5}^{2}}\left\{P^{\mu \nu}+\eta^{\mu \nu} \partial_{y}\left[e^{-2 \sigma} \partial_{y}\right]+2 r_{i} \delta\left(y-y_{i}\right) P^{\mu \nu}+\frac{1}{\xi} \partial^{\mu} \partial^{\nu}\right\} G_{\nu \alpha}\left(X, X^{\prime}\right) & =\delta_{\alpha}^{\mu} \delta\left(X-X^{\prime}\right) \\
\frac{1}{g_{5}^{2}}\left\{\partial^{2}+\xi \partial_{y}^{2} e^{-2 k y}\right\} G_{55}\left(X, X^{\prime}\right) & =\delta\left(X-X^{\prime}\right),
\end{aligned}
$$

where $P^{\mu \nu} \equiv \eta^{\mu \nu} \partial^{2}-\partial^{\mu} \partial^{\nu}$ and $r_{i} \delta\left(y-y_{i}\right)$ indicates the sum over both the UV and IR branes.

Due to the translational invariance of Eq. (3) along the four noncompact dimensions, it is convenient to work in mixed position and momentum space, defined by $G\left(p ; y, y^{\prime}\right)=$ $\int d^{4} x e^{i \eta_{\mu \nu} p^{\mu} x^{\nu}} G\left(x, y, y^{\prime}\right)$. The momentum defined in this way is a conserved quantity. However, it is important to keep in mind that it is not necessarily the physical momentum of the propagating field. Rather, from Eq. (3) it can be inferred that the momentum that an observer would measure when standing at $y$ is $p_{p h y s}=e^{k y} p$. In the following, we will be working with the "coordinate" momentum $p$, as is standard in the literature, with the understanding that $p$ is the momentum as measured by UV observers. Keeping the fifth coordinate explicit will also make the locality properties in $y$ manifest. We choose to work in Euclidean space, which will simplify the analysis later. Then the $G_{\mu \nu}\left(p ; y, y^{\prime}\right)$ propagator can be written as

$$
G_{\mu \nu}\left(p ; y, y^{\prime}\right)=\left(\eta_{\mu \nu}-\frac{p_{\mu} p_{\nu}}{p^{2}}\right) G_{p}\left(y, y^{\prime}\right)+\frac{p_{\mu} p_{\nu}}{p^{2}} G_{p}^{\prime}\left(y, y^{\prime}\right)
$$

where $G_{p}\left(y, y^{\prime}\right)$ and $G_{p}^{\prime}\left(y, y^{\prime}\right)$ satisfy

$$
\begin{aligned}
\frac{1}{g_{5}^{2}}\left\{\partial_{y}\left[e^{-2 \sigma} \partial_{y}\right]-p^{2}\left[1+2 r_{i} \delta\left(y-y_{i}\right)\right]\right\} G_{p}\left(y, y^{\prime}\right) & =\delta\left(y-y^{\prime}\right), \\
\frac{1}{g_{5}^{2}}\left\{\partial_{y}\left[e^{-2 \sigma} \partial_{y}\right]-\frac{p^{2}}{\xi}\right\} G_{p}^{\prime}\left(y, y^{\prime}\right) & =\delta\left(y-y^{\prime}\right) .
\end{aligned}
$$


The $\delta$-functions impose the following boundary conditions at $y=0, L$ :

$$
\begin{aligned}
& {\left[\partial_{y} G_{p}-r_{U V} p^{2} e^{2 \sigma} G_{p}\right]_{y=0}=0} \\
& {\left[\partial_{y} G_{p}+r_{I R} p^{2} e^{2 \sigma} G_{p}\right]_{y=L}=0}
\end{aligned}
$$

whereas $\partial_{y} G_{p}^{\prime}$ must vanish at the boundaries. At $y=y^{\prime}$, both $G_{p}$ and $G_{p}^{\prime}$ must be continuous and satisfy

$$
e^{-2 \sigma}\left[\left.\partial_{y} G_{p}^{(\prime)}\right|_{y=y^{\prime}+\epsilon}-\left.\partial_{y} G_{p}^{(\prime)}\right|_{y=y^{\prime}-\epsilon}\right]=g_{5}^{2},
$$

where $\epsilon \rightarrow 0^{+}$. The solutions can be written as

$$
G_{p}\left(y, y^{\prime}\right)=\frac{g_{5}^{2} e^{k\left(y+y^{\prime}\right)}}{k(A D-B C)}\left[A K_{1}\left(\frac{p}{k} e^{k y<}\right)+B I_{1}\left(\frac{p}{k} e^{k y<}\right)\right]\left[C K_{1}\left(\frac{p}{k} e^{k y>}\right)+D I_{1}\left(\frac{p}{k} e^{k y>}\right)\right]
$$

where $K_{\alpha}, I_{\alpha}$ are modified Bessel functions of order $\alpha, y_{<(>)}$are the smallest (largest) of $y, y^{\prime}$ and

$$
\begin{aligned}
A & =I_{0}\left(\frac{p}{k}\right)-p r_{U V} I_{1}\left(\frac{p}{k}\right) \\
B & =K_{0}\left(\frac{p}{k}\right)+p r_{U V} K_{1}\left(\frac{p}{k}\right) \\
C & =I_{0}\left(\frac{p}{k} e^{k L}\right)+p e^{k L} r_{I R} I_{1}\left(\frac{p}{k} e^{k L}\right) \\
D & =K_{0}\left(\frac{p}{k} e^{k L}\right)-p e^{k L} r_{I R} K_{1}\left(\frac{p}{k} e^{k L}\right) .
\end{aligned}
$$

The solution for $G_{p}^{\prime}$ takes the same form with $p \rightarrow p / \xi$ and $r_{U V}=r_{I R}=0$ in Eq. (14). The $G_{55}\left(p ; y, y^{\prime}\right)$ propagator is of the same form as $G_{p}^{\prime}$, but with second order Bessel functions and multiplied by $1 / \xi$. From these results we see how the transition into the unitary gauge, $\xi \rightarrow \infty$ (or $\mathcal{A}_{5}=0$ ) proceeds; $G_{55} \rightarrow 0$ and $G_{p}^{\prime} \rightarrow G_{0}$. In this gauge, all of the physics is contained in Eqs. (13) and (14).

To understand the physics contained in Eq. (13) it is instructive to analyze it in various limits. We define the following auxiliary function

$$
B\left(Y_{>}, Y_{<}, r\right)=\frac{1}{1+p r}\left\{\left[1+e^{-\frac{2 p}{k}\left(e^{k Y>}-e^{k Y}<\right)}\right]+p r\left[1-e^{-\frac{2 p}{k}\left(e^{k Y>}-e^{k Y}<\right)}\right]\right\},
$$

which, as the notation indicates, will be used only when $Y_{>}>Y_{<}$. In this case, $B\left(Y_{>}, Y_{<}, r\right)$ has a very simple behavior when the condition $(2 p / k) e^{k Y_{>}} \gg 1$ is satisfied: $B \approx 2 /(1+p r)$ when $Y_{<}$is (extremely) close to $Y_{>}$, and $B \approx 1$ otherwise. Specializing to the case of interest for addressing the hierarchy problem, where $e^{-k L} \ll 1$, we can write: 
i) For $p \gg k$,

$$
G_{p}\left(y, y^{\prime}\right) \sim-\frac{g_{5}^{2}}{2 p} e^{\frac{1}{2} k\left(y+y^{\prime}\right)} e^{-\frac{p}{k}\left(e^{k y>}-e^{k y<}\right)} B\left(y_{<}, 0, r_{U V}\right) B\left(L, y_{>}, e^{k L} r_{I R}\right) .
$$

ii) For $k \gg p \gg k e^{-k y<}$,

$$
G_{p}\left(y, y^{\prime}\right) \sim-\frac{g_{5}^{2}}{2 p} e^{\frac{1}{2} k\left(y+y^{\prime}\right)} e^{-\frac{p}{k}\left(e^{k y>}-e^{k y<}\right)} B\left(L, y_{>}, e^{k L} r_{I R}\right) .
$$

iii) For $k e^{-k y_{<}} \gg p \gg k e^{-k y>}$,

$$
G_{p}\left(y, y^{\prime}\right) \sim-\frac{g_{5}^{2}}{2 p^{2}} e^{\frac{1}{2} k y>} e^{-\frac{p}{k} e^{k y>}} \sqrt{\frac{\pi p}{2 k}} \frac{2 k+\left(e^{2 k y<}-1\right) p^{2} r_{U V}}{\ln (2 k / p)+k r_{U V}} B\left(L, y_{>}, e^{k L} r_{I R}\right) .
$$

iv) For $k e^{-k y>} \gg p \gg k e^{-k L}$,

$$
G_{p}\left(y, y^{\prime}\right) \sim-\frac{g_{5}^{2}}{2 p^{2}} \frac{2 k+\left(e^{2 k y<}-1\right) p^{2} r_{U V}}{\ln (2 k / p)+k r_{U V}} .
$$

v) For $p \ll k e^{-k L}$,

$$
G_{p}\left(y, y^{\prime}\right) \sim-\frac{g_{5}^{2}}{2 k p^{2}} \frac{\left[2 k+\left(e^{2 k y<}-1\right) p^{2} r_{U V}\right]\left[2 k+\left(e^{2 k L}-e^{2 k y>}\right) p^{2} r_{I R}\right]}{2 k\left(L+r_{U V}+r_{I R}\right)+e^{2 k L} p^{2} r_{U V} r_{I R}} .
$$

Several remarks are in order. Consider first the properties of the propagator when the kinetic terms vanish. If we are doing physics at a fixed location along the warped dimension (say, on one of the branes), it follows from the previous asymptotic forms that the propagator $G_{p}(y, y)$ changes from a four dimensional $\left(\sim 1 / p^{2}\right)$ to a five dimensional $(\sim 1 / p)$ behavior at a scale $p \sim k e^{-k y}$. The onset of the higher dimensional scaling indicates that the gauge theory ceases to be predictive when the external $4 \mathrm{~d}$ momenta are much larger than $k e^{-k y}$, and that it should be cut off at a scale $\Lambda e^{-k y}$, for some constant $\Lambda \gtrsim k$. Thus, a $y$-dependent cutoff on $p$ appears naturally in this language. ${ }^{2}$

For the consistency of this picture, it is important to note that, at energy scales above $k e^{-k y}$, the observables localized at $y$ are only sensitive to the physics of nearby points $y^{\prime}$,

\footnotetext{
${ }^{2}$ The $y$-dependence of the cutoff is simply a consequence of general covariance and the fact that AdS is homogeneous. The statement that the highest energy modes described by the effective theory have $p \sim \Lambda e^{-k y}$ translates in position space into minimum coordinate wavelengths of size $\Delta x \sim 1 / p \sim e^{k y} / \Lambda$. That is to say, the effective theory describes proper wavelengths larger than $\Delta s=e^{-k y} \Delta x \sim 1 / \Lambda$, independent of the position in the bulk. By a similar reasoning, one can see that the powers of the warp factors $e^{k y}$ in Eqs. (16)- 201) are also dictated by general covariance.
} 
such that $\exp \left(-p\left|e^{k y}-e^{k y^{\prime}}\right| / k\right) \sim 1$. Furthermore, case iii) shows that the contributions from far away points $y^{\prime}>y$ are exponentially suppressed for all momenta above the lower cutoff $\Lambda e^{-k y^{\prime}}$. Thus, the physics associated with some energy scale at $y$ is effectively shielded from contributions that would be outside the region of validity of the effective theory.

It is remarkable that the gauge field tree-level propagator with endpoints at $y$ exhibits a purely four-dimensional scaling at all scales below $k e^{-k y}$. This property together with the shielding discussed in the previous paragraph are an important ingredient in the understanding of the four-dimensional "running" of the gauge couplings that has been discussed recently [12, 13. In fact, if one defines the gauge coupling at $y$ at a scale $p$ via the two point gauge correlator with endpoints at $y$ and external momentum $p$, as advocated in [13], one can understand from the above discussion that, provided $p<k e^{-k y}$, all the propagators that are relevant in a loop calculation will exhibit a four dimensional scaling. Therefore, such momentum integrals will depend logarithmically on $p$, with no power-law sensitivity.

From the behavior of the five dimensional propagator $G_{p}\left(y, y^{\prime}\right)$, Eqs. (16) -(20), we can also quickly understand some of the effects induced by the presence of the local terms. Considering again coincident points $y=y^{\prime}$, we see that for $p \gg k e^{-k y}$, there are three different regions. Away from the boundaries we find, as before, the five dimensional scaling $G_{p}(y, y) \sim 1 / p$, and we conclude that the brane kinetic terms have an exponentially small effect (that does not show up at this order), as expected from locality. On the branes, however, we find $G_{p}(0,0) \sim$ $g_{5}^{2} /\left(p+p^{2} r_{U V}\right)$ and similarly for $G_{p}(L, L)$ with $e^{k L} r_{I R}$ in place of $r_{U V}$ and $g_{5}^{2} e^{k L}$ in place of $g_{5}^{2}$. This shows that, for brane observers, a four dimensional behavior is recovered for $p \gg 1 / r_{c}$ where $r_{c}=r_{U V}$ on the UV brane and $r_{c}=e^{k L} r_{I R}$ on the IR brane. Note that the IR crossover distance has been appropriately red-shifted as is generally expected for dimensionful parameters in the Randall-Sundrum setup.

We postpone a further analysis of the physics behind the behavior of the propagator when $r_{U V}$ and $r_{I R}$ are nonzero to the next section. Here we only note that, from a theoretical point of view, it is perfectly consistent to take the coefficients $r_{U V}, r_{I R}$ to be large in units of the fundamental length $1 / M$, as long as we include their effects exactly as has been done here. As we have seen, the previous exact propagator does not change the divergence structure of the $5 \mathrm{~d}$ theory, except on the branes, where it actually softens it. In particular, it is consistent to assume that all other couplings are small so that perturbation theory is valid, even if the brane kinetic terms are larger than their "natural" scale [14]. Nevertheless, when the gauge fields are identified with the carriers of the standard model interactions, one can derive some constraints 
on the size of the brane kinetic terms from the fact that the observed standard model gauge couplings are of order one. For simplicity, and because it is the case that will interest us in the phenomenological applications of section 5 , let us consider the case where $r_{U V}=0$. From Eq. (20), we see that the observed zero mode gauge coupling, $g_{0}$, is given by

$$
g_{0}^{2}=\frac{g_{5}^{2}}{L+r_{I R}}=\frac{g_{5}^{2}}{L}\left(1+\frac{r_{I R}}{L}\right)^{-1} .
$$

In the limit that $r_{I R} \gg L$, this gives $r_{I R} \simeq g_{5}^{2} / g_{0}^{2}$. This relation does not directly impose a bound on $r_{I R} / L$ which, as will be shown in the following sections is the most relevant quantity. However, if one assumes that the gauge observables at any given position $y$ are perturbative below the local "compactification scale" $k e^{-k y}$, as would be desirable from the point of view of gauge coupling unification, one has $g_{5}^{2} k \lesssim 16 \pi^{2}$, a four-dimensional loop factor (this can be inferred from Eq. (19) $)$. Under this assumption, it follows that $r_{I R} / L \lesssim 16 \pi^{2} /\left(g_{0}^{2} k L\right)$. Recalling now that a solution to the hierarchy problem requires $k L \sim 35$, we conclude that $r_{I R} / L \lesssim 5 / g_{0}^{2}$, which can be of order twenty for the electroweak interactions.

\section{Kaluza-Klein Decomposition}

From the point of view of an observer at low energies, the relevant description of the phenomenology is in terms of the Kaluza-Klein modes. Bulk gauge fields in warped backgrounds with transparent branes were considered in [15]. In order to elucidate the effects of the opaque branes, we begin with a brief review of some of the features of the transparent brane case.

\subsection{Review of the Transparent Brane Case}

We begin with the linearized 5d equation of motion for a bulk gauge field. From the action, Eq. (11), with $r_{i}=0$, we derive the equation for the $\lambda$ component of $A$,

$$
\partial_{\sigma} \partial^{\sigma} \mathcal{A}^{\lambda}-\partial^{\lambda}\left(\partial_{\sigma} \mathcal{A}^{\sigma}\right)-2 k e^{-2 k y}\left(\partial_{y} \mathcal{A}^{\lambda}\right)+e^{-2 k y}\left(\partial_{y}^{2} \mathcal{A}^{\lambda}\right)=0
$$

In order to determine the KK spectrum, we expand $\mathcal{A}(X)$ in wave functions,

$$
\mathcal{A}^{\lambda}(X)=\sum_{n} f_{n}(y) A_{n}^{\lambda}\left(x^{\mu}\right)
$$

and require the $A_{n}^{\lambda}$ to obey the $4 \mathrm{~d}$ equation of motion for a free massive gauge field,

$$
\partial_{\sigma} \partial^{\sigma} A_{n}^{\lambda}-\partial^{\lambda}\left(\partial_{\sigma} A_{n}^{\sigma}\right)-m_{n}^{2} A_{n}^{\lambda}=0
$$


This requires the wave functions to obey the equation,

$$
\left[\partial_{y}^{2}-2 k \partial_{y}+e^{2 k y} m_{n}^{2}\right] f_{n}(y)=0
$$

The solutions to this equation are Bessel functions,

$$
f_{n}(y)=\mathcal{N}_{m} e^{k|y|}\left\{J_{1}\left(\frac{m_{n}}{k} e^{k|y|}\right)+b Y_{1}\left(\frac{m_{n}}{k} e^{k|y|}\right)\right\}
$$

where $\mathcal{N}_{m}$ is an overall normalization factor, determined by requiring each KK mode to have canonically normalized kinetic terms,

$$
\begin{aligned}
\frac{1}{g_{5}^{2}} \int_{0}^{L} d y f_{n}(y) f_{m}(y) & =\delta_{n m} \\
\frac{1}{g_{5}^{2}} \int_{0}^{L} d y e^{-2 k y} f_{n}^{\prime}(y) f_{m}^{\prime}(y) & =m_{n}^{2} \delta_{n m} .
\end{aligned}
$$

and $b$ is a function of $m_{n}$, both of which are determined by boundary conditions below.

In order to find the masses, we impose boundary conditions that the first derivative of the wave functions be continuous at the $y=0$ and $y=L$ boundaries. This requires,

$$
b^{0}=-\frac{J_{0}\left(\frac{m_{n}}{k}\right)}{Y_{0}\left(\frac{m_{n}}{k}\right)} \quad, \quad b^{L}=-\frac{J_{0}\left(\frac{m_{n}}{k} e^{k L}\right)}{Y_{0}\left(\frac{m_{n}}{k} e^{k L}\right)} .
$$

Requiring both boundary conditions be satisfied simultaneously, $b^{0}=b^{L}$, provides a transcendental equation for the quantized masses. This equation may be solved numerically, resulting in masses, in units of $k e^{-k L}$, of 0 , and approximately 2.5, 5.6, 8.7, . [15], where the difference between two subsequent mass eigenvalues tends asymptotically to $\pi$.

The coupling of the $n$-th KK gauge boson mass eigenstate to matter on the brane at $y=L$ is equal to $f_{n}(L)$ times the appropriate charge of the matter field. For large values of $k L$, the KK modes couple universally (up to a sign) to fermions located on the IR brane at $y=L$, with a coupling $\sqrt{2 k L}$ larger than the zero mode's coupling (for $k L \simeq 35$, the coupling is therefore approximately 8.5 times larger than the zero mode coupling). The KK modes couple to fields on the UV brane at $y=0$ with couplings that vary with the KK mode number, and are typically suppressed compared to the zero mode coupling. This behavior may be seen in Fig. 1, for $r_{I R}=0$.

It is interesting to compare these results with the behavior of the five-dimensional propagator for two points located on either the IR or the UV brane. For $p \gg k e^{-k L}$, that is for momenta 
much larger than the mass of the lightest KK mode, the five dimensional propagator with endpoints on the IR brane reads

$$
\begin{aligned}
G_{p}(L, L) & =-\frac{g_{5}^{2}}{p} e^{k L} \\
& =-\frac{2 g_{5}^{2} k}{p^{2}} \frac{\pi}{2}\left(\frac{p}{\pi k e^{-k L}}\right) .
\end{aligned}
$$

Using the result that, for $p \gg \tilde{m} \equiv \pi k e^{-k L}$, one can approximate $\sum_{n}\left[1 /\left(p^{2}+(n \tilde{m})^{2}\right)\right] \simeq$ $\left(\pi / 2 p^{2}\right)(p / \tilde{m})$, Eq. (29) has a clear interpretation. The five dimensional behavior of the propagator is given by the sum over KK modes with a constant coupling $g_{n}^{2}=2 g_{5}^{2} k$ to the IR brane. Since the zero mode coupling is given by $g_{0}^{2}=g_{5}^{2} / L$, the relation between these couplings is the one described above.

On the UV brane, the propagator reaches a five dimensional behavior only at momenta much larger than $k$. Indeed, from Eq. (16) one obtains

$$
G_{p}(0,0)=-\frac{g_{5}^{2}}{p} .
$$

The comparison between Eqs. (29) and (30) suggests that those KK modes with masses larger than $k$ couple with a constant coupling $2 g_{5}^{2} k e^{-k L}$ to the UV brane. Observe that these couplings are exponentially suppressed with respect to the couplings on the IR brane. A numerical evaluation of the couplings of asymptotically large mass eigenstates for $k L \simeq 5$ confirms that this is indeed the case.

\subsection{The Opaque Brane Case}

Now we allow for opacity on both the IR and the UV branes, allowing $r_{I R} \neq 0, r_{U V} \neq 0$ in Eq. (11). In this case, the orthonormality conditions for the KK decomposition become,

$$
\begin{aligned}
\frac{1}{g_{5}^{2}} \int_{0}^{L} d y\left[1+2 r_{U V} \delta(y)+2 r_{I R} \delta(y-L)\right] f_{n}(y) f_{m}(y) & =\delta_{n m} \\
\frac{1}{g_{5}^{2}} \int_{0}^{L} d y e^{-2 k y} f_{n}^{\prime}(y) f_{m}^{\prime}(y) & =m_{n}^{2} \delta_{n m} .
\end{aligned}
$$

Eq. (31) reduces to Eq. (27) for $r_{U V}=r_{I R}=0$. These conditions may be simultaneously solved, as before, by imposing the condition that the $4 \mathrm{~d}$ gauge fields $A_{n}^{\lambda}\left(x^{\mu}\right)$ are on the mass shell. The resulting wave functions satisfy,

$$
\left[\partial_{y}^{2}-2 k \partial_{y}+e^{2 k y} m_{n}^{2}\left(1+2 r_{U V} \delta(y)+2 r_{I R} \delta(y-L)\right)\right] f_{n}(y)=0
$$


In fact, the introduction of opaque branes does not affect the bulk solution for the KK modes, as written in Eq. (26), but instead modifies the boundary conditions at $y=0$ and $y=L$ to reflect the discontinuity in the slope of the wave function. Thus, the new solutions have the same form, but with different $b$ 's,

$$
\begin{aligned}
b^{0} & =-\frac{J_{0}\left(\frac{m_{n}}{k}\right)+m_{n} r_{U V} J_{1}\left(\frac{m_{n}}{k}\right)}{Y_{0}\left(\frac{m_{n}}{k}\right)+m_{n} r_{U V} Y_{1}\left(\frac{m_{n}}{k}\right)}, \\
b^{L} & =-\frac{J_{0}\left(\frac{m_{n}}{k} e^{k L}\right)-m_{n} r_{I R} e^{k L} J_{1}\left(\frac{m_{n}}{k} e^{k L}\right)}{Y_{0}\left(\frac{m_{n}}{k} e^{k L}\right)-m_{n} r_{I R} e^{k L} Y_{1}\left(\frac{m_{n}}{k} e^{k L}\right)},
\end{aligned}
$$

indicating different admixture of the Bessel functions $J_{1}$ and $Y_{1}$ in the solutions. Once again, we determine the quantized masses by imposing the conditions on both boundaries, $b^{0}=b^{L}$. While there is no analytic way to solve for the masses, solutions may easily be obtained numerically.

Once the eigenmasses (and therefore $b$ ) have been found, we normalize the wave functions and determine the coupling to either brane-localized or bulk fields. The normalization condition to determine $\mathcal{N}_{n}$ in the presence of brane kinetic terms, Eq. (31), may be expressed as,

$$
\frac{1}{g_{5}^{2}}\left[r_{U V} f_{n}^{2}(0)+r_{I R} f_{n}^{2}(L)+\int_{0}^{L} d y f_{n}^{2}(y)\right]=1,
$$

where the explicit factors of two multiplying the $r_{i}$ brane terms that were introduced in Eq. (11) cancel as a result of our having considered only the physical space $0 \leq y \leq L$ and not the reflection, $y<0$.

To examine the couplings of the KK tower to various types of fields, either confined to branes or living in the bulk, consider some representative interaction terms in the $5 \mathrm{~d}$ theory,

$$
\begin{aligned}
\mathcal{L}_{\text {int }}=\int_{0}^{L} d y & \left\{\delta\left(y-y_{\psi}\right)\left[\bar{\psi} \mathcal{A}_{\mu} \gamma^{\mu} \psi\right]\right. \\
+ & \frac{1}{g_{5}^{2}}\left(1+2 r_{U V} \delta(y)+2 r_{I R} \delta(y-L)\right)\left[2\left(\partial_{\mu} \mathcal{A}_{\nu}^{a}-\partial_{\nu} \mathcal{A}_{\mu}^{a}\right) f^{a b c} \mathcal{A}_{b}^{\mu} \mathcal{A}_{c}^{\nu}\right] \\
& \left.+\frac{1}{g_{5}^{2}}\left(1+2 r_{U V} \delta(y)+2 r_{I R} \delta(y-L)\right)\left[f^{a b c} f^{a d e} \mathcal{A}_{b}^{\mu} \mathcal{A}_{c}^{\nu} \mathcal{A}_{\mu}^{d} \mathcal{A}_{\nu}^{e}\right]\right\},
\end{aligned}
$$

where we have taken fermions canonically normalized and confined to a brane located at $y=y_{\psi}$. The first term represents the gauge boson-fermion coupling and the latter two terms are the interactions among the bulk gauge fields for a non-Abelian theory. In order to derive the effective interactions between various KK modes, one inserts the KK decomposition into this equation. 
From our normalization convention for the $f_{n}(y)$, it follows that the coupling of brane fields localized at $y_{\psi}$ to the $n$th $\mathrm{KK}$ mode is

$$
g_{n}=f_{n}\left(y_{\psi}\right)
$$

Equation (32) always has a solution with zero mass and constant wave function. The constant wave function insures that the zero mode couples universally to all charged brane matter regardless of where it is localized, as required by the unbroken gauge invariance of the zero mode. The normalization of the zero mode in terms of $g_{5}, r_{I R}, r_{U V}$, and $L$ determines the zero mode gauge coupling in terms of the fundamental parameters,

$$
g_{0}=f_{0}(y)=\frac{g_{5}}{\sqrt{L+r_{I R}+r_{U V}}} .
$$

Since it is the coupling of the lightest mode which we identify at low energies with our four dimensional gauge interaction, we choose to present the couplings of the higher KK modes relative to the zero mode coupling.

Couplings of the higher KK modes of bulk fields are model-dependent, being given by integrals of products of several of the wave functions. For the three- and four-point gauge field vertices, we have,

$$
\begin{aligned}
g_{n m l} & =\frac{1}{g_{5}^{2}} \int_{0}^{L} d y\left(1+2 r_{U V} \delta(y)+2 r_{I R} \delta(y-L)\right) f_{n}(y) f_{m}(y) f_{l}(y) \\
g_{n m l k} & =\frac{1}{g_{5}^{2}} \int_{0}^{L} d y\left(1+2 r_{U V} \delta(y)+2 r_{I R} \delta(y-L)\right) f_{n}(y) f_{m}(y) f_{l}(y) f_{k}(y)
\end{aligned}
$$

between the $A_{n}-A_{m}-A_{l}$ and $A_{n}-A_{m}-A_{l}-A_{k}$ modes, respectively. For simplicity of notation we have suppressed the vector and color indices, but these are readily restored. For the vertices involving the zero mode, the results are much simpler, since the zero mode wave function is independent of $y$. In the three-point vertex, we find that the zero mode has constant coupling $g_{0} \delta_{m n}$ with all other $\mathrm{KK}$ mode pairs. In the four-point vertex, setting $l=k=0$ results in a vertex factor $g_{0}^{2} \delta_{m n}$. Together, these results demonstrate the fact that the zero mode gauge field's couplings take a universal form as dictated by its unbroken gauge invariance, resulting in the same coupling to both bulk and brane fields. These results are the same as in the flat brane scenario studied in [9].

\subsection{Opaque IR Brane}

To begin with, we consider the case where $r_{I R} \neq 0$ but $r_{U V}=0$. We fix $k L=34.5$ as a typical value which generates approximately the correct hierarchy between the Planck and weak scales. 
In Figure 1, we present the masses of the four lowest modes as a function of $r_{I R} k$. As the brane kinetic term increases, the KK mode masses decrease, each one approaching an asymptotic value. The features are qualitatively similar to the flat space case [9].

Also plotted in Figure 1 are the couplings of each mode to fields localized in either the UV or the IR branes. In agreement with the intuition that the localized kinetic term expels the higher KK modes from the brane, we see that the modes decouple from the IR brane, with the heavier modes decoupling more quickly. (In contrast, the couplings to the UV brane fields increase with larger $r_{I R}$.) This behavior can also be understood from the point of view of quantum mechanics. For sufficiently large spatial momenta, the particle loses information about the extension of the extra dimensions and the physics on the brane becomes determined by the value of the local gauge coupling. The larger the value of $r$ and the larger the spatial momentum, the more striking this phenomenon must be. In the large $r$ limit, the particle propagator $G_{p}(L, L)$ should recover its four dimensional behavior, and its interactions must be equivalent to the one of a particle with gauge coupling equal to the local brane one. This is only possible if the higher KK modes, which dominate the large momentum behavior of the propagator, decouple from the brane, a behavior that is shown in Fig. 11.

Some analytical understanding of the behavior of couplings and masses is provided by the analysis of the five dimensional propagator for $r_{I R} \neq 0$ and $r_{U V}=0$. For $p \gg k e^{-k L}$ the propagator, Eq. (16), at $y=y^{\prime}=L$ reduces to

$$
G_{p}(L, L) \simeq-\frac{g_{5}^{2} e^{k L}}{p\left(1+p r_{I R} e^{k L}\right)} .
$$

Therefore, for values of $k r_{I R} \gtrsim 1$, one recovers a four dimensional behavior in this energy regime,

$$
G_{p}(L, L) \simeq-\frac{g_{5}^{2}}{p^{2} r_{I R}}
$$

This expression can be compared with the one obtained for momenta $p \ll k e^{-k L}$ from Eq. (201),

$$
G_{p}(L, L) \sim-\frac{g_{5}^{2}}{p^{2}\left(L+r_{I R}\right)},
$$

which describes the zero mode with coupling $g_{0}=g_{5} / \sqrt{L+r_{I R}}$. The simplest interpretation of the transition from Eq. (42) to Eq. (43) is that there is a single massive mode with mass of order $k e^{-k L}$, that couples to the brane with strength

$$
g_{1}=\sqrt{\frac{L}{r_{I R}}} g_{0},
$$



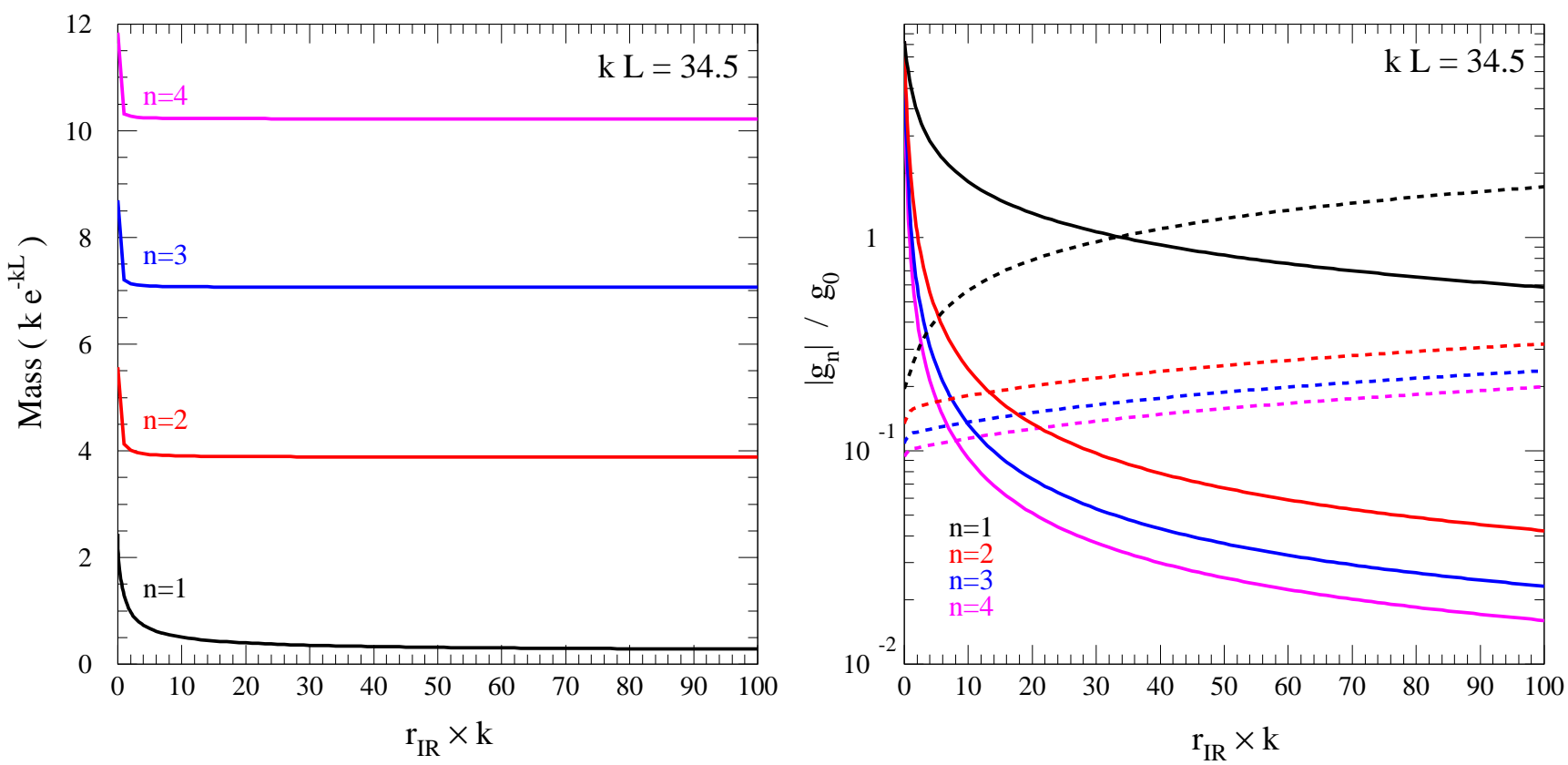

Figure 1: The $n=1,2,3,4$ (bottom to top) KK mode masses in units of $k e^{-k L}$ and couplings to IR brane fields (solid lines) and UV brane fields (dashed lines) relative to the zero mode coupling for the case in which the IR brane is opaque, as a function of the opacity $r_{I R} k$.

while the other modes decouple. ${ }^{3}$ We also see that when $r_{I R} \gg L$ then $g_{1} \ll g_{0}$, and therefore, in the large brane kinetic term limit, only the zero mode couples to the brane with coupling $g_{0}^{2} \approx g_{I R}^{2}=g_{5}^{2} / r_{I R}$. This result agrees with the numerical results displayed in Fig. 11.

\subsection{Two Opaque Branes}

The physics in the two opaque brane scenario is somewhat different from the one brane case. In analogy with the case of flat extra dimensions [9] for values of $r_{I R}$ and $r_{U V}$ large compared to both $L$ and $1 / k$, the physics at each brane must be determined by the local couplings and, in the asymptotic limit of $r_{I R}, r_{U V} \rightarrow \infty$ an observer on either the UV or IR brane must be insensitive to the presence of the extra dimensions, including the other brane. This can only be true if, in this limit, two massless modes appear, and appropriate linear combinations of them couple to one of the branes with a strength given by the local coupling, while decoupling from the other brane and vice versa. Since for any finite value of $r_{I R}$ and $r_{U V}$ there is only one zero

\footnotetext{
${ }^{3}$ For example, for $k L=34.5$, as in Fig. $1, m_{1} \rightarrow 0.24 k e^{-k L}$ as $k r_{I R} \rightarrow \infty$.
} 
mode, what should happen is that the mass of the first KK mode tends to zero as the local brane terms increase, while keeping a relevant coupling to the IR and UV branes.

This behavior was already apparent from the form of the five dimensional propagator for large values of the local brane kinetic terms. Consider the propagator, $G_{p}\left(y, y^{\prime}\right)$ for $p \ll k e^{-k L}$, Eq. (20), for $y=y^{\prime}=L$ :

$$
\begin{aligned}
G_{p}(L, L) & \sim-\frac{g_{5}^{2}}{p^{2}} \frac{2 k+e^{2 k L} p^{2} r_{U V}}{2 k\left(L+r_{U V}+r_{I R}\right)+e^{2 k L} p^{2} r_{U V} r_{I R}} \\
& =-\frac{g_{5}^{2}}{p^{2}\left(L+r_{U V}+r_{I R}\right)}-\frac{g_{5}^{2}\left(L+r_{U V}\right)}{r_{I R}\left(L+r_{U V}+r_{I R}\right)\left[p^{2}+m_{1}^{2}\right]} .
\end{aligned}
$$

The first term is the contribution of the massless zero mode, with coupling $g_{0}$ as defined in Eq. (38). The second term in Eq. (45) describes an additional mode of mass $m_{1} \equiv \gamma k e^{-k L}$, where

$$
\gamma=\sqrt{\frac{2\left(L+r_{U V}+r_{I R}\right)}{k r_{U V} r_{I R}}} .
$$

Provided that $\gamma \ll 1$, the propagator, Eq. (45), shows that there is a light mode (in addition to the zero mode), whose coupling is given by

$$
g_{1}=\sqrt{\frac{L+r_{U V}}{r_{I R}}} g_{0},
$$

which generalizes Eq. (44) for nonzero $r_{U V}$.

We see that, when $r_{U V}=r_{I R} \gg L$, the light mode couples with the same strength as the zero mode to fields localized on the IR brane. Notice also that, similarly to the case of vanishing $r_{U V}$, when $r_{I R} \gg r_{U V}, L$, the light mode decouples from the IR brane.

In the numerical analysis, for simplicity, we consider the case $r=r_{I R}=r_{U V}$. In Figure 2 we show the couplings to brane fields localized on the IR and UV branes. The first mode mass goes to zero and its coupling becomes equal (and opposite in sign in the case of UV brane fields) to the zero mode. This agrees with the limit $r \rightarrow \infty$ in which bulk propagation switches off and we are left with two brane gauge theories which do not interact with each other. The higher modes decouple from both branes. Note that the couplings to the UV brane grow slightly as $r$ increases, and then begin to fall off again as the higher modes decouple.

\section{Localized Higgs Effect}

In order to describe the electroweak theory and address the hierarchy problem, the Higgs responsible for electroweak symmetry-breaking (EWSB) must be localized on the IR brane 

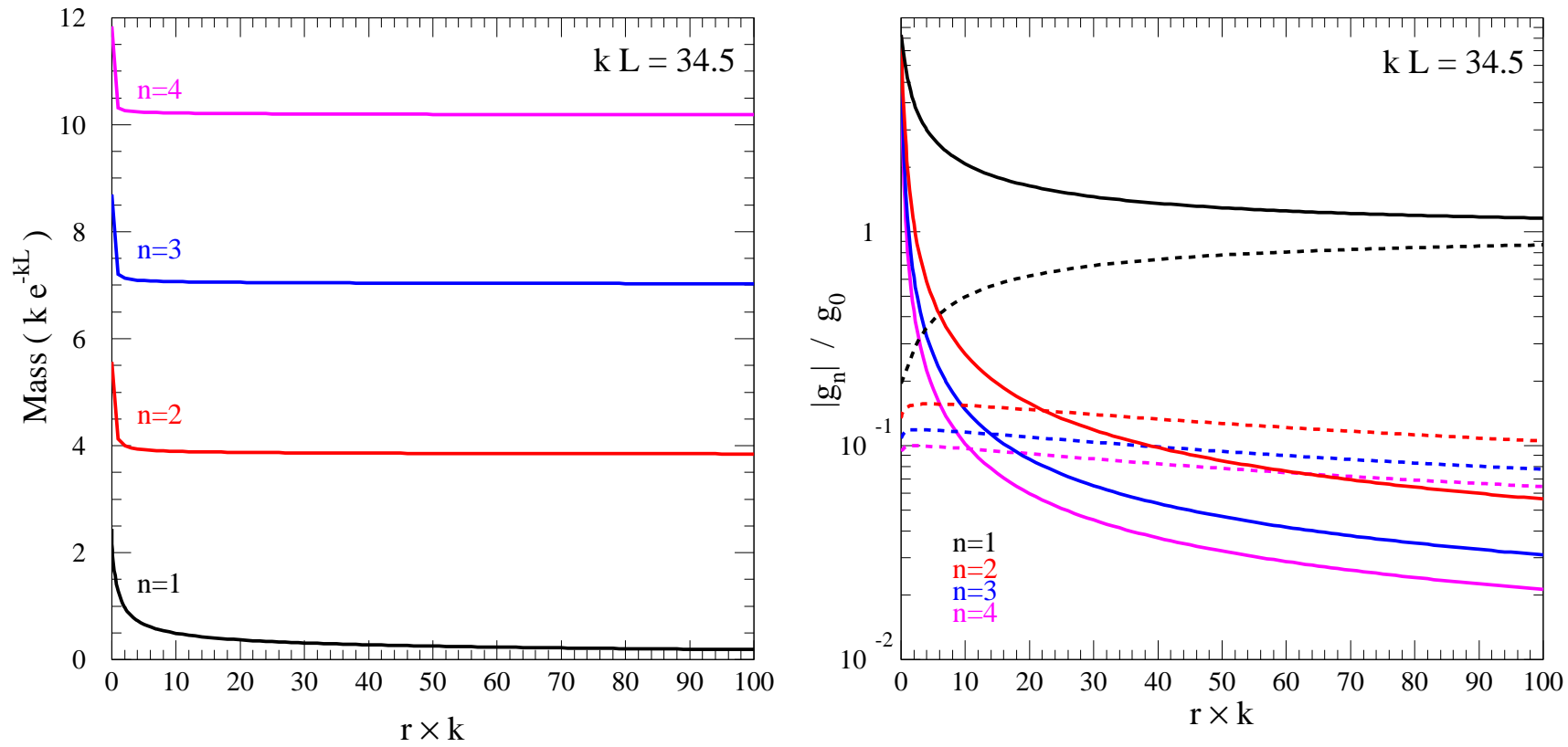

Figure 2: The $n=1,2,3,4$ (bottom to top) KK mode masses in units of $k e^{-k L}$ and couplings to IR brane fields (solid lines) and UV brane fields (dashed lines) relative to the zero mode coupling for the case in which both the IR and UV branes are opaque, as a function of the common opacity $r \times k$.

with action, ${ }^{4}$

$$
-\int d^{4} x d y \sqrt{-g} 2 \delta(y-L)\left\{\left(D_{\mu} H\right)^{\dagger} D^{\mu} H+\lambda\left(|H|^{2}-\frac{1}{2} v^{2}\right)^{2}\right\},
$$

where $D^{\mu}=\partial^{\mu}-i A^{\mu}$ is the usual gauge-covariant derivative. In the low energy effective theory (after rescaling the Higgs kinetic term to canonical normalization) this results in,

$$
-\int d^{4} x\left\{\eta^{\mu \nu}\left(D_{\mu} H\right)^{\dagger} D_{\nu} H+\lambda\left(|H|^{2}-\frac{1}{2} v^{2} e^{-2 k L}\right)^{2}\right\},
$$

with the EWSB VEV red-shifted to $\tilde{v}=e^{-k L} v$. As mentioned above, a natural solution to the hierarchy problem is obtained for values of $k L \simeq 34.5$. In $5 \mathrm{~d}$ language and working in $4 \mathrm{~d}$ unitary gauge, the localized VEV results in a gauge boson mass which is itself localized on the IR brane,

$$
-\frac{1}{2} \int d^{4} x d y 2 \delta(y-L) \tilde{v}^{2} \eta^{\mu \nu} \mathcal{A}_{\mu} \mathcal{A}_{\nu}
$$

\footnotetext{
${ }^{4}$ The minus sign in front of the scalar kinetic term is due to our metric signature convention, Eq. (3). The factor of two in front of the $\delta$-function has the same origin as for the localized terms in Eq. (1).
} 
It is therefore interesting to analyze the effects on the gauge field propagation induced by the presence of a Higgs VEV on the IR brane. In fact, the localized Higgs VEV bears a certain resemblance to the local gauge kinetic terms, and it can be analyzed using similar techniques. For the purposes of this section, we consider a simple gauge group. We discuss the subtleties of the $S U(2)_{L} \times U(1)_{Y}$ theory in section 5 .

The localized mass only affects the boundary condition to be imposed at $y=L$. Comparing Eqs. (11) and (150) we see that, for example, Eq. (111) is modified to

$$
\left[\partial_{y} G_{p}+e^{2 \sigma}\left(r_{I R} p^{2}+g_{5}^{2} \tilde{v}^{2}\right) G_{p}\right]_{y=L}=0 .
$$

Therefore, we can obtain $G_{p}\left(y, y^{\prime}\right)$ from Eqs. (13) and (14) by making the replacement

$$
r_{I R} \rightarrow r_{I R}+\frac{g_{5}^{2} \tilde{v}^{2}}{p^{2}}
$$

The conditions for a diagonal KK decomposition may be expressed as

$$
\begin{aligned}
\frac{1}{g_{5}^{2}} \int_{0}^{L} d y f_{n}(y) f_{m}(y)\left[1+2 r_{U V} \delta(y)+2 r_{I R} \delta(y-L)\right] & =\delta_{n m}, \\
\frac{1}{g_{5}^{2}} \int_{0}^{L} d y e^{-2 k y}\left\{f_{n}^{\prime}(y) f_{m}^{\prime}(y)+2 g_{5}^{2} v^{2} \delta(y-L) f_{n}(y) f_{m}(y)\right\} & =m_{n}^{2} \delta_{n m} .
\end{aligned}
$$

Once again the KK mode wave functions can be found by requiring each mode to satisfy the free field equation of motion for a massive vector field. The KK masses are determined as before, by imposing $b^{0}=b^{L}$, where now the coefficient $b^{L}$ in Eq. (26), determined by the boundary condition at $y=L$, can be simply obtained from Eq. (34) by the replacement (152) with $p^{2}=-m_{n}^{2}$ :

$$
b^{L}=-\frac{J_{0}\left(\frac{m_{n}}{k} e^{k L}\right)-\left[m_{n} r_{I R}-g_{5}^{2} \tilde{v}^{2} / m_{n}\right] e^{k L} J_{1}\left(\frac{m_{n}}{k} e^{k L}\right)}{Y_{0}\left(\frac{m_{n}}{k} e^{k L}\right)-\left[m_{n} r_{I R}-g_{5}^{2} \tilde{v}^{2} / m_{n}\right] e^{k L} Y_{1}\left(\frac{m_{n}}{k} e^{k L}\right)} .
$$

We will be interested in the case that $r_{U V}$ vanishes (or is small). In this case, $b^{0}$ is given by

$$
b^{0}=-\frac{J_{0}\left(\frac{m_{n}}{k}\right)}{Y_{0}\left(\frac{m_{n}}{k}\right)} .
$$

\subsection{Transparent IR Brane}

Let us start with the case $r_{I R}=0$. A first intuitive phenomenon that occurs is that, due to energy considerations, the presence of the $\mathrm{VEV}$ tries to induce a repulsion of the gauge field 

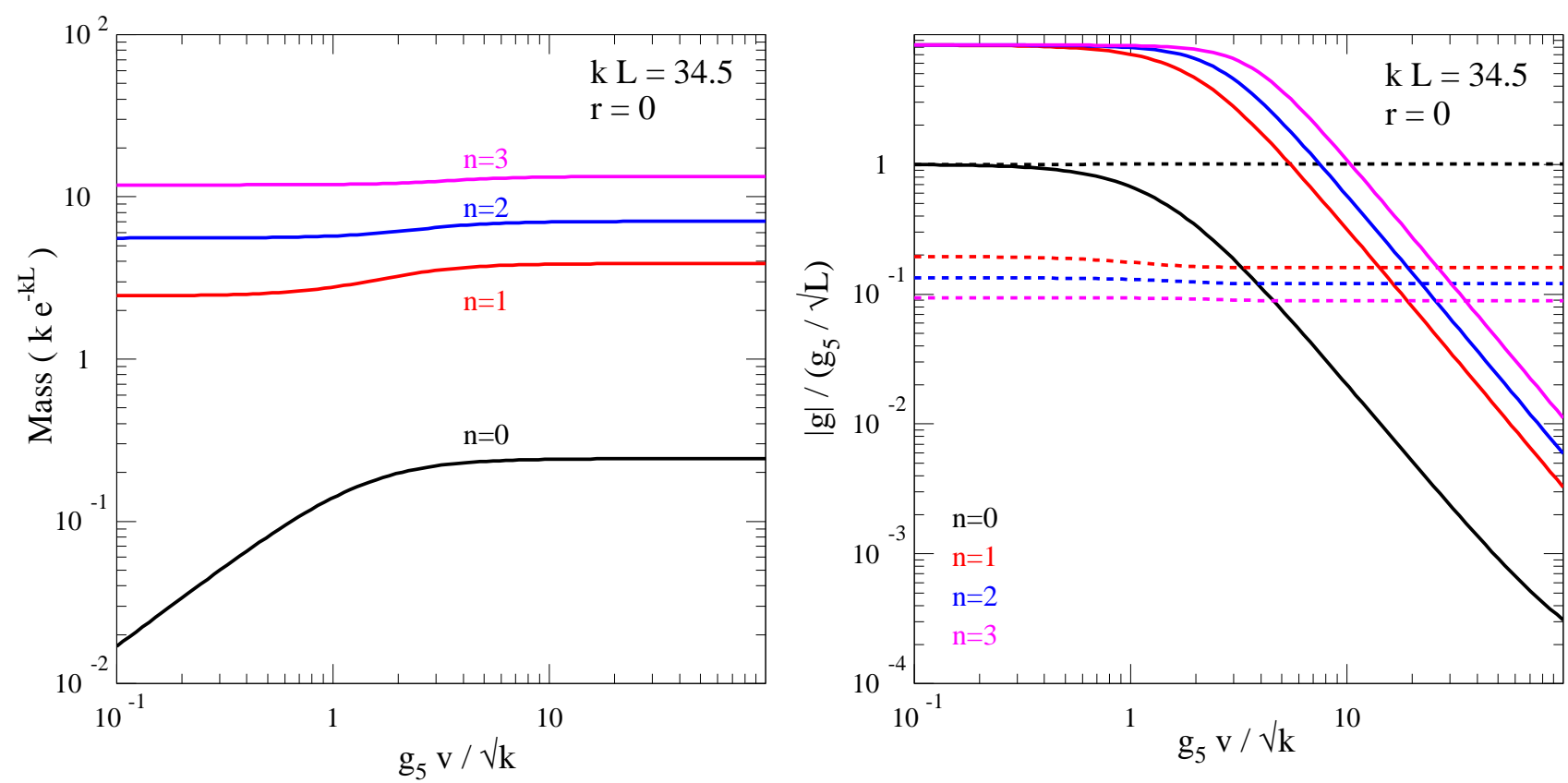

Figure 3: The $n=0,1,2,3$ (bottom to top) KK mode masses in units of $k e^{-k L}$ and couplings to IR brane fields (solid lines) and UV brane fields (dashed lines) relative to the bulk coupling $\left(g_{5} / \sqrt{L}\right)$ for the case in which a Higgs develops a VEV $v$ on the IR brane, and the local kinetic terms are zero, as a function of the $\mathrm{VEV}, v$

from the brane location. This is balanced by the cost in energy associated with a non-vanishing $y$-derivative. In a KK decomposition, this produces mixing between the whole tower of $\mathrm{KK}$ modes, resulting in a deformation of the five dimensional wave functions, including the zero mode, which is no longer absolutely flat. ${ }^{5}$ When $v \ll k$, the cost associated with the deformed wave function overshadows the localized mass induced by the Higgs, and the zero mode remains approximately flat with mass given by $g_{5} \tilde{v} / \sqrt{L}$.

However, when $v \gg k$, the cost associated with the derivative is small compared to that associated with the brane mass. In that case the light KK modes are expelled from the brane. The repulsion of the gauge field from the IR brane has as an immediate consequence that all the KK mode gauge couplings, as well as the zero mode one, tend to small values. For large values of $v / k$, the ratio of the KK mode couplings to the zero mode tends to a constant that becomes larger than the value $\sqrt{2 k L}$ obtained for $v=0$. The second effect is that, due to the decoupling

\footnotetext{
${ }^{5}$ For simplicity, we continue to refer to the lightest (would-be zero) mode as the zero mode, despite the fact that its mass is no longer zero.
} 
of the gauge field from the IR brane, the masses of the zero mode (and light KK modes for sufficiently large values of $v / k$ ) are no longer governed by the Higgs VEV. This behavior is apparent in Figure 3 where one sees that for $v<k$ the mass of the zero mode increases linearly with $v$, with value $g_{5} \tilde{v} / \sqrt{L}$. For $v \gtrsim k$, the mass of the lightest mode becomes more and more insensitive to $v$ as the mode bends away from the brane. Also shown in Figure 3 are the couplings of the four lowest modes to the IR and UV branes relative to the zero mode coupling in the absence of a localized Higgs VEV,$g_{5} / \sqrt{L}$. We see the behavior inferred above: for $v \ll k$ the coupling is close to $g_{5} / \sqrt{L}$, while for $v \gtrsim k$ the KK modes are repelled from the brane. This results in the higher KK modes more and more strongly coupled to the IR brane compared to the zero mode. In this case, the theory becomes strongly coupled more quickly than it would have in the absence of the VEV, and the KK modes have a dramatic effect on the phenomenology.

\subsection{Opaque IR brane}

The situation in the case of a local gauge kinetic term on the IR brane is somewhat different. This is due to the fact that at sufficiently large momenta the physics should be dominated by the local brane term and therefore for large values of $r_{I R}$ compared to $L$ and $1 / k$ there should be a mode with coupling to the IR brane given by the local gauge coupling and mass given approximately by this coupling times the VEV of the Higgs field. All other modes should decouple from the IR brane, and their masses need not be correlated with $v$.

We can make these observations more concrete by studying the propagator with endpoints on the IR brane. Its limiting forms in the high energy and low energy regimes are, respectively,

$$
\begin{array}{rlrl}
G_{p}(L, L) & \sim-\frac{g_{5}^{2}}{p e^{-k L}\left(1+p r_{I R} e^{k L}\right)+g_{5}^{2} \tilde{v}^{2}} & & p \gg k e^{-k L} \\
G_{p}(L, L) & \sim-\frac{g_{5}^{2}}{p^{2}\left(L+r_{I R}\right)+g_{5}^{2} \tilde{v}^{2}} & p \ll k e^{-k L} .
\end{array}
$$

We see that for sufficiently large momenta, $p r_{I R} e^{k L} \gg 1$, the propagator Eq. (56) reduces to $G_{p}(L, L) \sim-g_{I R}^{2} /\left(p^{2}+g_{I R}^{2} \tilde{v}^{2}\right)$, where $g_{I R}^{2}=g_{5}^{2} / r_{I R}$. Thus, when $g_{I R} v \gg \max \left(k, 1 / r_{I R}\right)$, the propagator describes a single four dimensional state of mass $g_{I R} \tilde{v}$ (up to corrections of order $\left.k /\left(g_{I R} v\right)\right)$. It is also clear that the energy at which this behavior sets in is lower for larger $r_{I R} k$. In fact, when $r_{I R} \gg L(\gg 1 / k)$ the low energy propagator Eq. (57) takes exactly the previous form, so that the full propagator reduces to the propagator of a single four dimensional state with mass $g_{I R} \tilde{v}$ and coupling $g_{I R}$. 
The above described behavior is illustrated in Fig. 4 from a KK point of view, where we plot the masses and couplings (now relative to $g_{5} \sqrt{k}$ ) of the four lightest modes for two different values of $r_{I R}$, as a function of $v / k$. The mass spectrum exhibits a level-crossing phenomenon, more pronounced for $r_{I R} k=10$ than for $r_{I R} k=1$, where each mode successively "takes a turn" feeling the Higgs effect. For small $v$, it is the zero mode whose mass grows linearly with $v$, whereas at $v / k \sim 1$ the zero mode mass becomes insensitive to $v$, and it is the first KK mode mass which grows linearly. This pattern repeats; as each mode becomes insensitive to $v$, the one above it becomes sensitive and grows linearly with $v$. For large $v / k$, the transition points occur in intervals of approximately $r_{I R} k$, with effective coupling approximately $g_{5} / \sqrt{r_{I R}}$, apart from the zero mode whose coupling is always given by $g_{5} / \sqrt{L+r_{I R}}$.

For the phenomenological applications to be discussed in section 5, we will be interested mostly in the case where $\tilde{v} \ll k e^{-k L}$. It is clear from Eq. (57) that, for $p \ll k e^{-k L}$, there is a single state with coupling and mass given by $g_{0} \simeq g_{5} / \sqrt{L+r_{I R}}$ and $m_{0} \simeq g_{0} \tilde{v}$, up to corrections of order $g_{5}^{2} v^{2} / k$. It will be useful to have the next order corrections to the above parameters. We find

$$
\begin{aligned}
& m_{0}=\frac{g_{5} \tilde{v}}{\sqrt{L+r_{I R}}}\left[1-\eta\left(\frac{g_{5}^{2} v^{2}}{k}\right)+\mathcal{O}\left(\frac{g_{5}^{2} v^{2}}{k}\right)^{2}\right], \\
& g_{0}=\frac{g_{5}}{\sqrt{L+r_{I R}}}\left[1-2 \eta\left(\frac{g_{5}^{2} v^{2}}{k}\right)+\mathcal{O}\left(\frac{g_{5}^{2} v^{2}}{k}\right)^{2}\right],
\end{aligned}
$$

where

$$
\eta=\frac{2 k^{2} L^{2}-2 k L+1}{8 k^{2}\left(L+r_{I R}\right)^{2}}
$$

We observe that when $r_{I R} \gg L$ then $\eta \sim L^{2} / r_{I R}^{2}$, which shows explicitly that, provided $g_{5}^{2} v^{2} / k$ is small, in the large $r_{I R}$ limit the standard relations among $\tilde{v}, g_{0}$ and $m_{0}$ are recovered.

\subsection{Two Opaque Branes}

For the sake of completeness we now consider the case where both $r_{U V}$ and $r_{I R}$ are turned on. The IR brane propagator has the following limits for arbitrary values of $r_{U V}, r_{I R}$ and $g_{5} \tilde{v}$ :

$$
\begin{aligned}
& G_{p}(L, L) \sim-\frac{g_{5}^{2}}{p e^{-k L}\left(1+p r_{I R} e^{k L}\right)+g_{5}^{2} \tilde{v}^{2}} \quad p \gg k e^{-k L} \\
& G_{p}(L, L) \sim-g_{5}^{2}\left[p^{2}\left(r_{I R}+\frac{2 k\left(L+r_{U V}\right)}{2 k+e^{2 k L} p^{2} r_{U V}}\right)+g_{5}^{2} \tilde{v}^{2}\right]^{-1} \quad p \ll k e^{-k L} .
\end{aligned}
$$





Figure 4: The $n=0,1,2,3$ (bottom to top) KK mode masses in units of $k e^{-k L}$ and couplings to IR brane fields (relative to $g_{5} \sqrt{k}$ ) for the case in which a Higgs develops a VEV on the IR brane, and the local kinetic terms are $r_{I R} k=1$ (red dashed curves) or $r_{I R} k=10$ (black solid curves), as a function of the VEV, $g_{5} v / k$. The dotted lines in the coupling figure indicate the value of the local brane coupling (relative to $g_{5} \sqrt{k}$ ) corresponding to the appropriate value of $r_{I R}$.

We see that, as required by locality, when $p \gg k e^{-k L}$, the IR propagator depends only on $r_{I R}$, not on $r_{U V}$ which is localized far away. Thus, the high energy properties are the same as when $r_{U V}=0$, as discussed in the previous subsection: for sufficiently large momenta, IR observers see a single four dimensional state of mass $g_{I R} \tilde{v}$ and coupling $g_{I R}$.

The low energy limit is more complicated and depends on the relative size of the various localized parameters. First, inspecting the coefficient of the $p^{2}$ term in Eq. (62), we see that when $p \ll k e^{-k L}$ there is a qualitative difference depending on the size of $\gamma$ as defined in Eq. (46). When $\gamma \gg 1$, as is the case when either brane localized kinetic term vanishes, the propagator is just

$$
G_{p}(L, L) \sim-\frac{g_{0}^{2}}{p^{2}+g_{0}^{2} \tilde{v}^{2}},
$$

where $g_{0}^{2}$ was defined in Eq. (38). The physics is qualitatively similar to the case where $r_{U V}=0$.

The low energy physics is considerably richer when both localized kinetic terms are present 
and $\gamma \ll 1$. In this case we have to be more careful about the size of the Higgs VEV relative to other scales. In fact, compared to the analysis of subsection 3.4, the introduction of the Higgs VEV introduces two new relevant scales: $g_{I R} \tilde{v}$ and $g_{0} \tilde{v}$, where $g_{I R}^{2}=g_{5}^{2} / r_{I R}$ and $g_{0}$ is given in Eq. (38). These two scales can be very different if there is a hierarchy between $r_{U V}$ and $r_{I R}$. For simplicity, we will analyze here the case where $g_{0} \sim g_{I R}$. We find three regions according to the size of $g_{I R} v$. When $g_{I R} v \gg k$ there are no light states in the theory and we get an effective contact term interaction $G_{p}(L, L) \sim-1 / \tilde{v}^{2}$. When $\gamma k \ll g_{I R} v \ll k$ we find

$$
G_{p}(L, L) \sim\left\{\begin{array}{cc}
-\frac{g_{I R}^{2}}{p^{2}} & g_{I R} \tilde{v} \ll p \ll k e^{-k L} \\
-\frac{1}{\tilde{v}^{2}} & p \ll g_{I R} \tilde{v},
\end{array}\right.
$$

which shows that, below the compactification scale $k e^{-k L}$, there is a single state with mass $g_{I R} \tilde{v}$, and with coupling $g_{I R}$. Finally, when $g_{I R} \tilde{v} \ll \gamma k$, we conclude from

$$
G_{p}(L, L) \sim\left\{\begin{array}{rrrl}
-\frac{g_{I R}^{2}}{p^{2}} & \gamma k e^{-k L} & \ll p \ll k e^{-k L} \\
-\frac{g_{0}^{2}}{p^{2}+g_{0}^{2} \widetilde{v}^{2}} & p \ll \gamma k e^{-k L} .
\end{array}\right.
$$

that there are two light states below $k e^{-k L}$. The lightest one has mass $g_{0} \tilde{v}$ and coupling $g_{0}$. The heavier one has a mass of order $\gamma k e^{-k L}$ and coupling $g_{1}$ as given in Eq. (47).

\section{Electroweak Theory and Phenomenology}

We now turn to the electroweak theory $S U(2)_{L} \times U(1)_{Y}$, and realistic phenomenology. We assume that the Higgs and all fermions are confined to the IR brane, but the gauge fields are allowed to propagate in the bulk. For simplicity, we treat the UV brane as transparent: $r_{U V}=0$. After EWSB, the relevant terms in the 5 d Lagrangian are thus,

$$
\begin{aligned}
\mathcal{L}_{E W}^{5}=\sqrt{-g}\{ & -\frac{1}{4 g_{5}^{2}} \mathcal{W}_{M N} \mathcal{W}^{M N}\left(1+2 r_{2} \delta(y-L)\right)-\frac{1}{4 g_{5}^{\prime 2}} \mathcal{B}_{M N} \mathcal{B}^{M N}\left(1+2 r_{1} \delta(y-L)\right) \\
& \left.-v^{2} \delta(y-L)\left[\mathcal{W}_{M}^{1} \mathcal{W}_{1}^{M}+\mathcal{W}_{M}^{2} \mathcal{W}_{2}^{M}+\left(\mathcal{W}_{M}^{3}-\mathcal{B}_{M}\right)\left(\mathcal{W}_{3}^{M}-\mathcal{B}^{M}\right)\right]\right\},
\end{aligned}
$$

where the $\mathcal{W}_{M}^{(1,2,3)}$ are the three $S U(2)_{L}$ gauge bosons (with bulk coupling $g_{5}$ ), $\mathcal{B}_{M}$ is the $U(1)_{Y}$ gauge boson (with bulk coupling $g_{5}^{\prime}$ ), and $\mathcal{W}_{M N}$ and $\mathcal{B}_{M N}$ are the respective field strength tensors. $r_{1}$ and $r_{2}$ are the IR brane gauge kinetic terms for the $U(1)_{Y}$ and $S U(2)_{L}$ gauge groups. 
In Ref. [4, which addressed the localized VEV effects, but treated the brane as transparent, the situation was analyzed in the limit $v \ll k$ (for which the symmetry-breaking effects of section 4 can be treated as a perturbation) by introducing the gauge rotations which diagonalize the masses in terms of the bulk couplings, $s \equiv g_{5}^{\prime} / \sqrt{g_{5}^{2}+g_{5}^{\prime 2}}$. Then, in the basis,

$$
\mathcal{W}_{\mu}^{3}=c^{2} \mathcal{Z}_{\mu}+\mathcal{A}_{\mu} \quad, \quad \mathcal{B}_{\mu}=-s^{2} \mathcal{Z}_{\mu}+\mathcal{A}_{\mu}
$$

the KK towers for the photon $(A)$ and $Z$ decouple from each other. For $r_{1} \neq r_{2}$, this choice is not particularly convenient, because although it does decouple the KK tower of the photon and $Z$ with respect to the bulk terms, they remain mixed together by the brane terms. Thus we consider the simplified case, $r_{1}=r_{2}$, for which the same rotation diagonalizes both terms. While there is no reason why $r_{1}=r_{2}$ should hold, and in fact since they renormalize differently, they will certainly differ at different energy scales, it does simply illustrate the effects of the brane kinetic terms on the phenomenological picture.

When $r_{1}=r_{2}=r$, the field redefinitions of Eq. (67) diagonalize the Lagrangian, resulting in,

$$
\begin{aligned}
\mathcal{L}_{E W}^{5}= & \sqrt{-g}\left\{-\frac{s^{2}}{2 e_{5}^{2}} \mathcal{W}_{M N}^{+} \mathcal{W}_{-}^{M N}[1+2 r \delta(y-L)]-\frac{1}{4 e_{5}^{2}} \mathcal{F}_{M N} \mathcal{F}^{M N}[1+2 r \delta(y-L)]\right. \\
& \left.-\frac{s^{2} c^{2}}{4 e_{5}^{2}} \mathcal{Z}_{M N} \mathcal{Z}^{M N}[1+2 r \delta(y-L)]-2 v^{2} \delta(y-L)\left(\mathcal{W}_{M}^{+} \mathcal{W}_{-}^{M}+\frac{1}{2} \mathcal{Z}_{M} \mathcal{Z}^{M}\right)\right\},
\end{aligned}
$$

where we have introduced $1 / e_{5}^{2}=1 / g_{5}^{2}+1 / g_{5}^{\prime 2}$, the $5 \mathrm{~d}$ photon coupling.

We can now make contact with the results of section 4 Clearly the zero mode of the photon is associated with the gauge interactions we see at low energies. The weak gauge bosons are somewhat more subtle. In section 4 we saw that for $r \ll 1 / k$ the first few KK modes couple a factor of order 10 (or more for large $v / k$ ) more strongly to the IR brane than the zero mode, and have approximately equal spacing. This suggests that for a viable phenomenological picture, it is the zero modes that should be associated with the weak gauge bosons observed in experiments, with the higher KK modes suitably heavy such that they evade current experimental limits.

However, for large $r$, there is only ever one gauge boson with relevant gauge coupling to the fermions. Provided the lighter modes are sufficiently weakly coupled, they could have escaped detection up until now. Thus, for $r \sim 1 / k$ we have a choice as to which mode plays the role of the observed weak bosons, at least for some range of parameters. For simplicity, we will restrict our attention to the simpler case in which we associate the known weak interactions with the zero modes of the $\mathcal{W}$ and $\mathcal{Z}$ fields, and leave the more exotic case in which we may actually be identifying the higher KK modes as the mediators of the weak force for future work. 


\subsection{Matching to the Effective Theory}

In the Standard Model the physics predominantly depends on three parameters of the electroweak theory, $e, \sin ^{2} \theta_{W}$, and $G_{\mu}$. The accuracy of the data is such that loop-level effects are important, introducing a strong dependence on the top mass $\left(m_{t}\right)$, and relevant dependence on the strong coupling at the $Z$ pole $\left(\alpha_{S}\right)$ and Higgs mass $\left(m_{h}\right)$. The usual procedure is to use the three most precisely measured quantities, the Fermi constant from muon decay $\left(G_{\mu}\right)$, the mass of the $Z$ boson $\left(M_{Z}\right)$, and the electromagnetic coupling at the $Z$-pole $\left(\alpha_{Z}\right)$, to fix the three tree-level parameters, and then to combine the sum of the precision data in a fit to either $m_{h}$ (and $m_{t}$ and $\alpha_{S}$ ), or, allowing for nonstandard corrections to the weak boson self-energies (oblique corrections), to fit the oblique parameters $S, T$, and $U[16]$.

This procedure is not completely appropriate whenever there are important non-oblique corrections which directly modify the fermion couplings, or which modify the self-interactions of the gauge bosons beyond what is contained in the oblique parameters. However, it is still sensible whenever the oblique corrections capture the dominant new physics contributions. In the specific case of the RS model with bulk gauge bosons, one immediately encounters a problem related to non-oblique corrections. The muon decay now proceeds through the entire tower of KK modes of the $W$. This was handled in Refs. [3, 4, 17, ${ }^{6}$ by introducing an additional fit parameter $V$ which measures the amount of contribution to $G_{\mu}$ from the higher KK modes relative to the zero mode. However, as we will see below, we will find it more convenient to avoid introducing $V$, and instead define effective parameters, $S, T$, and $U$, which in practice take into account all relevant oblique and non-oblique corrections necessary to describe the Z-pole precision electroweak observables and the $\mathrm{W}$ mass.

At tree level, our model point is specified in terms of the Lagrangian in Eq. (68) by the six quantities $e_{5}, s, v, r, L$, and $k$. $k$ can be taken to be the 5 d Planck scale, and thus may be thought of as setting the overall dimensionful scale. We must identify the $4 \mathrm{~d}$ quantities $e$, $\sin ^{2} \theta_{W}$, and effective $4 \mathrm{~d}$ Higgs VEV $\tilde{v}$ from the measurements of $\alpha_{Z}, M_{Z}$, and $G_{\mu}$. This will determine three of our six parameters in terms of the other three. We represent this freedom by treating $r$ and $L$ as the free parameters, and use the input data to specify $e_{5}, s$, and $v$ in terms of them.

Applying the results of section 4, we derive the effective Lagrangian for the zero modes,

$$
-\frac{1}{2} W_{\mu \nu}^{+} W_{-}^{\mu \nu}-\frac{1}{4} Z_{\mu \nu} Z^{\mu \nu}-\frac{1}{4} F_{\mu \nu} F^{\mu \nu}-m_{W}^{2} W_{\mu}^{+} W_{-}^{\mu}-\frac{m_{Z}^{2}}{2} Z_{\mu} Z^{\mu}
$$

\footnotetext{
${ }^{6}$ See also Ref. [6], and Ref. [5] for a description of bulk fermion effects.
} 


$$
+\frac{1}{\sqrt{2}} f_{W}\left(\bar{\psi} \gamma^{\mu} T_{+} \psi W_{\mu}^{+}+\bar{\psi} \gamma^{\mu} T_{-} \psi W_{\mu}^{-}\right)+f_{Z} \bar{\psi} \gamma^{\mu}\left(T_{3}-s^{2} Q\right) \psi Z_{\mu}+f_{A} \bar{\psi} \gamma^{\mu} Q \psi A_{\mu},
$$

where $f_{W}, f_{Z}$ and $f_{A}$ are the $W, Z$ and photon zero mode wave functions evaluated at the IR brane, $y=L, \psi$ stands for the brane-localized fermions, $T_{ \pm}, T_{3}$ are the relevant weak isospin matrices and $Q$ is the electric charge. Note also that here and below, $4 \mathrm{~d}$ indices are raised and lowered by the canonically normalized Lorentz metric, $\eta_{\mu \nu}$. The quantities $m_{W}$ and $m_{Z}$ are determined numerically for a given choice of $v, e_{5}, s, r, L$, and $k$, as in Fig. 固. Note that this implies that these quantities have no direct relation to those which one would have expected in the SM.

Since the photon experiences no symmetry breaking, the zero mode wave function is flat, and thus we can identify the 4 d electromagnetic coupling $e$ with,

$$
e \equiv f_{A}=\frac{e_{5}}{\sqrt{L+r}} .
$$

This allows us to reproduce the electromagnetic coupling $\alpha_{Z}^{-1}=128.92(3)$. The muon decay constant $G_{\mu}$ should be identified with the (full) $W$ boson at zero momentum transfer. Thus, it implicitly contains the effects of the entire KK tower. From Eq. (57) we see that at zero momentum transfer, the tree-level gauge boson propagator reduces to the very simple form,

$$
-G\left(p^{2}=0 ; L, L\right)=\frac{f_{W}^{2}}{m_{W}^{2}}+\sum_{n \neq 0} \frac{f_{W_{n}}^{2}}{m_{W_{n}}^{2}}=\frac{1}{\tilde{v}^{2}}=4 \sqrt{2} G_{\mu},
$$

which implies that fixing $G_{\mu}$ to its experimentally measured value of $G_{\mu}=1.16639(1) \times 10^{-5}$ $\mathrm{GeV}^{-2}$ determines $\tilde{v} \simeq 123 \mathrm{GeV}$. This result for the propagator is exact at $p^{2}=0$ and includes the sum of the contributions of all the KK modes of the charged weak gauge bosons.

The final quantity we would like to use to determine the input parameters is the $Z$ mass. Having already determined $e_{5}$ and $v$ (as functions of $r$ and $L$ ), we can accomplish this by adjusting $s$ to the value which produces the correct $m_{Z}$. We find this value numerically as in section 4 adjusting the quantity $v e_{5} / s c$ until the mass of the lowest mode is the $Z$ boson mass measured in experiments, $M_{Z}=91.1875(21)$. The advantage of matching directly to $\alpha_{Z}, M_{Z}$, and $G_{\mu}$ is that, with appropriate definitions, we can carry over the established machinery for electroweak fits, and use the usual bounds on $S, T$, and $U$ to directly constrain the RS model, without refitting the data (see below).

It remains to determine the effect on precision observables as a function of $r$ and $L$, which allows us to determine the region of $r$ and $L$ consistent with experimental data. Since the gauge 
boson interactions with the fermions are affected in a universal way, most of the effects may be captured by effective $S, T$ and $U$ parameters. The primary remaining non-oblique effect is the coupling strengths of the self-interactions of the zero mode $W$ 's and $Z$. These depend on integrals over $y$ of three or four gauge boson wave functions. Thus, while they are determined from the $f(y)$, they are not related to them in a simple way. However, as these interactions are only mildly constrained by LEP-2 data [18, the resulting constraints are very weak.

We will find it convenient to rescale the zero mode wave functions,

$$
\begin{aligned}
f_{A} & =\frac{e_{5}}{\sqrt{L+r}} \hat{f}_{A} \\
f_{Z} & =\frac{e_{5}}{s c \sqrt{L+r}} \hat{f}_{Z}=\sqrt{g^{2}+g^{\prime 2}} \hat{f}_{Z} \\
f_{W} & =\frac{e_{5}}{s \sqrt{L+r}} \hat{f}_{W}=g \hat{f}_{W}
\end{aligned}
$$

for which the deviation from the tree-level SM predictions appears as a deviation of $\hat{f}$ from unity. Note that given our matching prescription we have $\hat{f}_{A}=1$. We have defined $g$ and $g^{\prime}$ with respect to $s$ and $c$, the sine and cosine of the gauge rotation angle defined above Eq. (67). This angle is different from the weak mixing angle implicit from our choice of input parameters,

$$
s_{0}^{2} c_{0}^{2}=\frac{\pi \alpha_{Z}}{\sqrt{2} G_{\mu} m_{Z}^{2}} .
$$

In order to make contact with the oblique parameters, we further rescale the fermion interactions to unity, resulting in the self-energy part of the effective Lagrangian taking the form,

$$
\begin{aligned}
& -\frac{1}{2 g^{2} \hat{f}_{W}^{2}} W_{\mu \nu}^{+} W_{-}^{\mu \nu}-\frac{1}{4\left(g^{2}+g^{2}\right) \hat{f}_{Z}^{2}} Z_{\mu \nu} Z^{\mu \nu}-\frac{1}{4 e^{2}} F_{\mu \nu} F^{\mu \nu} \\
& -\frac{m_{W}^{2}}{g^{2} \hat{f}_{W}^{2}} W_{\mu}^{+} W_{-}^{\mu}-\frac{m_{Z}^{2}}{2\left(g^{2}+g^{\prime 2}\right) \hat{f}_{Z}^{2}} Z_{\mu} Z^{\mu} .
\end{aligned}
$$

\subsection{The Effective $S, T$, and $U$ Parameters}

The tree-level contributions to $S, T$, and $U$ can be simply determined by matching the effective Lagrangian for the zero modes, Eq. (77) to a generic effective Lagrangian including a parameterization of the oblique corrections,

$$
\begin{aligned}
\mathcal{L}= & -\frac{1}{2 g^{2}}\left(1-\Pi_{W W}^{\prime}\right) W_{\mu \nu}^{+} W_{-}^{\mu \nu}-\frac{1}{4\left(g^{2}+g^{\prime 2}\right)}\left(1-\Pi_{Z Z}^{\prime}\right) Z_{\mu \nu} Z^{\mu \nu} \\
& -\frac{1}{4 e^{2}}\left(1-\Pi_{\gamma \gamma}^{\prime}\right) F_{\mu \nu} F^{\mu \nu}-\frac{s c}{2 e^{2}} \Pi_{\gamma Z}^{\prime} F^{\mu \nu} Z_{\mu \nu}-\left(\widetilde{v}^{2}+\frac{1}{g^{2}} \Pi_{W W}(0)\right) W_{\mu}^{+} W_{-}^{\mu}
\end{aligned}
$$




$$
\begin{aligned}
& -\frac{1}{2}\left(\widetilde{v}^{2}+\frac{1}{\left(g^{2}+g^{\prime 2}\right)} \Pi_{Z Z}(0)\right) Z_{\mu} Z^{\mu} \\
& +\frac{1}{\sqrt{2}}\left(\bar{\psi} \gamma^{\mu} T_{+} \psi W_{\mu}^{+}+\bar{\psi} \gamma^{\mu} T_{-} \psi W_{\mu}^{-}\right)+\bar{\psi} \gamma^{\mu}\left(T_{3}-s^{2} Q\right) \psi Z_{\mu}+\bar{\psi} \gamma^{\mu} Q \psi A_{\mu},
\end{aligned}
$$

where $\widetilde{v}$ plays the role of the Higgs VEV extracted from $G_{\mu}$, Eq . (72). We have followed Ref. [4] and written these in standard self-energy notation, despite the fact that the $5 \mathrm{~d}$ contributions are in fact tree level. The ADS/CFT correspondance suggests that the $5 \mathrm{~d}$ theory is dual to a $4 \mathrm{~d}$ theory of walking Technicolor [19. Thus, the tree level $5 \mathrm{~d}$ contributions correspond to the loop-level corrections in the dual theory [4. Matching Eqs. (77) and (78), we obtain,

$$
\begin{aligned}
\frac{\bar{\Pi}_{W W}^{\prime}}{g^{2}} & =\bar{\Pi}_{11}^{\prime}=\frac{1}{g^{2}}\left(1-\frac{1}{\hat{f}_{W}^{2}}\right) \\
\frac{\bar{\Pi}_{Z Z}^{\prime}}{\left(g^{2}+{g^{\prime 2}}^{2}\right)} & =\bar{\Pi}_{33}^{\prime}=\frac{1}{\left(g^{2}+g^{\prime 2}\right)}\left(1-\frac{1}{\hat{f}_{Z}^{2}}\right) \\
\bar{\Pi}_{3 Q}^{\prime} & =\bar{\Pi}_{Q Q}^{\prime}=0 \\
\frac{\bar{\Pi}_{W W}(0)}{g^{2}} & =\bar{\Pi}_{11}(0)=\frac{m_{W}^{2}}{g^{2} \hat{f}_{W}^{2}}-\widetilde{v}^{2} \\
\frac{\bar{\Pi}_{Z Z}(0)}{\left(g^{2}+g^{\prime 2}\right)} & =\bar{\Pi}_{33}(0)=\frac{m_{Z}^{2}}{\left(g^{2}+g^{\prime 2}\right) \hat{f}_{Z}^{2}}-\widetilde{v}^{2},
\end{aligned}
$$

where the $\bar{\Pi}$ refer to the fact that these are only the tree-level self-energy contributions from the extra-dimensional effects. The full $\Pi$ will also include both loop-level SM contributions, and also loop-level extra dimensional ones.

The usual oblique parameters $S, T$, and $U$ are defined in terms of the self-energies by,

$$
\begin{aligned}
S & \equiv 16 \pi\left(\Pi_{33}^{\prime}-\Pi_{3 Q}^{\prime}\right) \\
T & \equiv \frac{4 \pi}{s^{2} c^{2} M_{Z}^{2}}\left(\Pi_{11}(0)-\Pi_{33}(0)\right) \\
U & \equiv 16 \pi\left(\Pi_{11}^{\prime}-\Pi_{33}^{\prime}\right)
\end{aligned}
$$

where $M_{Z}$ is the $Z$ mass from the SM relations in terms of $e, s_{0}$ and $\tilde{v}$. Given our matching conditions, $m_{Z}=M_{Z}$. Thus, we derive the tree level $5 \mathrm{~d}$ contributions to the usual $S, T$, and U,

$$
\bar{S}=\frac{4 s^{2} c^{2}}{\alpha}\left(1-\frac{1}{\hat{f}_{Z}^{2}}\right)
$$




$$
\begin{aligned}
\bar{T} & =\frac{1}{\alpha}\left(\frac{m_{W}^{2}}{c^{2} m_{Z}^{2} \hat{f}_{W}^{2}}-\frac{1}{\hat{f}_{Z}^{2}}\right) \\
\bar{U} & =\frac{4 s^{2}}{\alpha}\left[1-\frac{1}{\hat{f}_{W}^{2}}-c^{2}\left(1-\frac{1}{\hat{f}_{Z}^{2}}\right)\right]
\end{aligned}
$$

where once again, the barred quantities indicate that these are only the contributions from the tree level extra-dimensional effects.

At the end of subsection 4.2, we found approximate expressions for the mass and coupling to IR fields of the zero mode gauge bosons, that are valid when $\epsilon \equiv g_{5}^{2} v^{2} / k \ll 1$ (see Eqs. (58) and (59) ), where $g_{5}$ stands for the appropriate gauge coupling ( $g_{5}$ for the charged $W$ bosons and $\sqrt{g_{5}^{2}+g_{5}^{\prime 2}}$ for the $Z$ ). It will be useful to have analytical expression for $S, T$ and $U$ to the same order. In our approach we determine the sine of the rotation angle between the gauge and mass eigenbasis, $s$, by requiring that the $Z$ mass is reproduced. This angle is in general different from the standard model value, but when $\epsilon \ll 1$ we expect them to differ by order $\epsilon$. Using Eq. (58) with the general $g_{5}$ of section 4 replaced by $e_{5} /(s c)$ we have

$$
m_{Z}=\frac{e \tilde{v}}{s c}(1-\eta \epsilon+\cdots) \equiv \frac{e \tilde{v}}{s_{0} c_{0}},
$$

where $\eta$ was defined in Eq. (60) and $\epsilon=e_{5}^{2} v^{2} /\left(s^{2} c^{2} k\right) \approx e_{5}^{2} v^{2} /\left(s_{0}^{2} c_{0}^{2} k\right)$. The above relation gives

$$
s=s_{0}\left(1-\frac{c_{0}^{2}}{c_{0}^{2}-s_{0}^{2}} \eta \epsilon+\cdots\right)
$$

where

$$
\eta \epsilon=\frac{2 k^{2} L^{2}-2 k L+1}{8 k\left(L+r_{I R}\right)} \frac{e^{2} v^{2}}{s_{0}^{2} c_{0}^{2} k^{2}} .
$$

We can also write $M_{W}, \hat{f}_{Z}(L)$ and $\hat{f}_{W}(L)$ as:

$$
\begin{aligned}
m_{W} & =\frac{e \tilde{v}}{s}\left[1-c^{2} \eta \epsilon+\mathcal{O}\left(\epsilon^{2}\right)\right]=\frac{e \tilde{v}}{s_{0}}\left[1+\frac{2 s_{0}^{2} c_{0}^{2}}{c_{0}^{2}-s_{0}^{2}} \eta \epsilon+\mathcal{O}\left(\epsilon^{2}\right)\right], \\
\hat{f}_{Z}(L) & =1-2 \eta \epsilon+\mathcal{O}\left(\epsilon^{2}\right), \\
\hat{f}_{W}(L) & =1-2 c_{0}^{2} \eta \epsilon+\mathcal{O}\left(\epsilon^{2}\right) .
\end{aligned}
$$

Plugging these expressions in Eqs. (87)-(89), we find

$$
\begin{aligned}
\bar{S} & =-\frac{16 s_{0}^{2} c_{0}^{2}}{\alpha} \eta \epsilon+\mathcal{O}\left(\epsilon^{2}\right), \\
\bar{T} & =-\frac{2 s_{0}^{2}}{\alpha} \eta \epsilon+\mathcal{O}\left(\epsilon^{2}\right), \\
\bar{U} & =\mathcal{O}\left(\epsilon^{2}\right) .
\end{aligned}
$$


Due to the presence of non-oblique corrections associated with heavy KK modes, the $S$, $T$ and $U$ parameters defined above are not sufficient to describe the precision electroweak observables. However, as observed in Ref. 4], the Z-pole precision observables may be properly described by the introduction of an effective parameter, $T_{\text {eff }}$, which includes the non-oblique corrections to the weak mixing angle,

$$
s^{2}-s_{0}^{2}=\frac{\alpha}{c^{2}-s^{2}}\left(\frac{1}{4} S-s^{2} c^{2} T_{\mathrm{eff}}\right)
$$

where $T_{\text {eff }}$ is given by

$$
T_{\text {eff }}=T+\Delta T
$$

and

$$
\Delta T=-\frac{1}{\alpha} \frac{\delta G_{\mu}}{G_{\mu}}=-\frac{1}{\alpha} \sum_{n \neq 0}\left(\frac{f_{W_{n}}^{2}}{f_{W}^{2}}\right)\left(\frac{m_{W}^{2}}{m_{W_{n}}^{2}}\right)=-\frac{1}{\alpha}\left(\frac{m_{W}^{2}}{\tilde{v}^{2} f_{W}^{2}}-1\right)=-\frac{2 c_{0}^{2}}{\alpha} \eta \epsilon+\mathcal{O}\left(\epsilon^{2}\right),
$$

where $\delta G_{\mu}$ is the non-oblique contribution to the muon decay constant due to the exchange of KK modes. With this definition of $T_{\text {eff }}$, we can recover Eq. (90), using Eqs. (92) and (94). Note that in the RS theory, the bare parameter $s$ coincides with the Kennedy-Lynn running coupling $s_{*}[20$.

The non-oblique corrections to $G_{\mu}$ affect also the expression of $m_{W} / m_{Z}$, but the relative coefficient between the oblique and non-oblique corrections encoded in $T_{\text {eff }}$ is different from the one appearing in $s^{2}$. Therefore, to properly parameterize $m_{W}^{2} / m_{Z}^{2}$ in terms of effective parameters $S_{\text {eff }}=S, T_{\text {eff }}$ and $U_{\text {eff }}$,

$$
\frac{m_{W}^{2}}{m_{Z}^{2}}-c_{0}^{2}=\frac{\alpha c^{2}}{c^{2}-s^{2}}\left(-\frac{1}{2} S_{\mathrm{eff}}+c^{2} T_{\mathrm{eff}}+\frac{c^{2}-s^{2}}{4 s^{2}} U_{\mathrm{eff}}\right)
$$

one can introduce

$$
U_{\text {eff }}=U-4 s^{2} \Delta T .
$$

Observe that the above expressions, Eqs. (98) and (101) reproduce Eqs. (90) and (92), respectively. $^{7}$

The above parameterization serves to describe all Z-pole observables as well as the $W$ mass. Following Ref. [21, we shall use a fit to these observables to place limits upon the free parameters of our theory.

\footnotetext{
${ }^{7}$ The parameterization for $m_{W}^{2} / m_{Z}^{2}$ in terms of the effective parameters $S_{\text {eff }}, T_{\text {eff }}$ and $U_{\text {eff }}$ differs from the one presented in the appendix of Ref. [4] by the additional contribution from $U_{\text {eff }}$.
} 
In terms of the expansion in $\epsilon$, we find that the tree-level five-dimensional contributions, including the non-oblique corrections to $G_{\mu}$, can be given by

$$
\begin{aligned}
& \bar{S}_{\mathrm{eff}}=-\frac{16 s_{0}^{2} c_{0}^{2}}{\alpha} \eta \epsilon+\ldots \approx-366 \eta \epsilon, \\
& \bar{T}_{\mathrm{eff}}=-\frac{2}{\alpha} \eta \epsilon+\ldots \approx-258 \eta \epsilon, \\
& \bar{U}_{\mathrm{eff}}=\frac{8 s_{0}^{2} c_{0}^{2}}{\alpha} \eta \epsilon+\ldots \approx 183 \eta \epsilon,
\end{aligned}
$$

We see that $\bar{S}_{\text {eff }}$ and $\bar{T}_{\text {eff }}$ are negative while $\bar{U}_{\text {eff }}$ is positive (and not small).

Before closing this section, let us stress that, following Ref. 4, we are ignoring loop-level contributions to the precision electroweak observables, including those induced by a potentially light radion. The computations of Ref. 22 indicate that, unless there is strong mixing between the radion and the Higgs field [23], these corrections are small and thus may be typically neglected. We have also checked that for the parameters that give a good description to the data, the effect of the tree-level neutral KK mode interactions in the Z-pole observables is unobservably small.

\subsection{Comparison with Data}

The SM with a light Higgs, with mass of about $120 \mathrm{GeV}$, provides an excellent description of the precision electroweak observables measured at the LEP, Tevatron and SLD colliders. While $\sin ^{2} \theta_{\text {eff }}$ plays a key role in the determination of the above quoted Higgs mass range, there is a discrepancy of more than about 3 $\sigma$ 's between the value of the weak mixing angle extracted from the lepton asymmetries and the one extracted from the hadron asymmetries. A good fit to the hadron asymmetries, which are dominated by the forward-backward asymmetry of the $b$-quark, $A_{F B}^{b}$, tends to require much larger values of the Higgs mass. On the other hand, a good fit to the lepton asymmetries leads to the preference of a Higgs mass value of about $50 \mathrm{GeV}[24$, well below the present direct search limit. In fact, the internal consistency of the precision electroweak data is dramatically improved by the exclusion of the hadron asymmetries: While the fit to the combined data from these colliders has a confidence of about 4\% [24, 21], it increases to more than $30 \%$ once the hadronic asymmetries are excluded.

This suggests that, in order to improve the consistency of the precision electroweak data

either one must postulate an error in the experimental determination of $A_{F B}^{b}$ and invoke new physics to raise the Higgs mass above the direct search limits [21], or one must introduce 
new physics that directly modifies the couplings of the bottom quark in order to restore the consistency of the measurements [25].

The RS model does not discriminate between the leptons and quarks, and thus this second option is denied to us. Below, we will consider the fits to precision electroweak parameters $S$, $T$, and $U$ both with and without $A_{F B}^{b}$. While we do not advocate ignoring $A_{F B}^{b}$ in general, it is interesting to see that the RS model with bulk gauge bosons can in fact lead to a remarkable improvement of the description of the electroweak observables once the hadron asymmetries are ignored, for a Higgs mass $m_{h}$ well above the experimental limits.

We use the $S, T$, and $U$ fits of [21], which, for $m_{t}=174.3 \mathrm{GeV}$ yield ${ }^{8}$ (removing $A_{F B}^{b}$ from the fit),

$$
\begin{aligned}
& S=-0.14 \pm 0.12 \\
& T=-0.08 \pm 0.13 \\
& U=0.20 \pm 0.14
\end{aligned}
$$

or (keeping $A_{F B}^{b}$ in the fit),

$$
\begin{aligned}
& S=0.00 \pm 0.11 \\
& T=-0.03 \pm 0.13 \\
& U=0.27 \pm 0.14 \text {. }
\end{aligned}
$$

We compute the full $S_{\text {eff }}, T_{\text {eff }}$, and $U_{\text {eff }}$ as the sum of the extra dimensional contributions, Eqs. (103)-(105), and also the contributions from the Higgs [16],

$$
\begin{aligned}
S_{H} & \simeq \frac{1}{12 \pi} \log \left(\frac{m_{h}^{2}}{m_{r e f}^{2}}\right) \\
T_{H} & \simeq-\frac{3}{16 \pi c_{0}^{2}} \log \left(\frac{m_{h}^{2}}{m_{r e f}^{2}}\right) \\
U_{H} & \simeq 0
\end{aligned}
$$

where $m_{\text {ref }}$ is a reference Higgs mass, chosen for the fits of Eqs. (106) and (107) to be $113 \mathrm{GeV}$.

We scan the parameter space of $L$ and $r$ for two fixed values of $m_{h}=115$ and $200 \mathrm{GeV}$, and identify the regions of $r$ and $L$ which are consistent with Eq. (106) or (107) at $1 \sigma, 2 \sigma$, and $3 \sigma$. We define consistency at the $n \sigma$ level to mean that all three oblique parameters predicted

\footnotetext{
${ }^{8}$ These fits do not differ very substantially from i.e., those obtained in [26].
} 

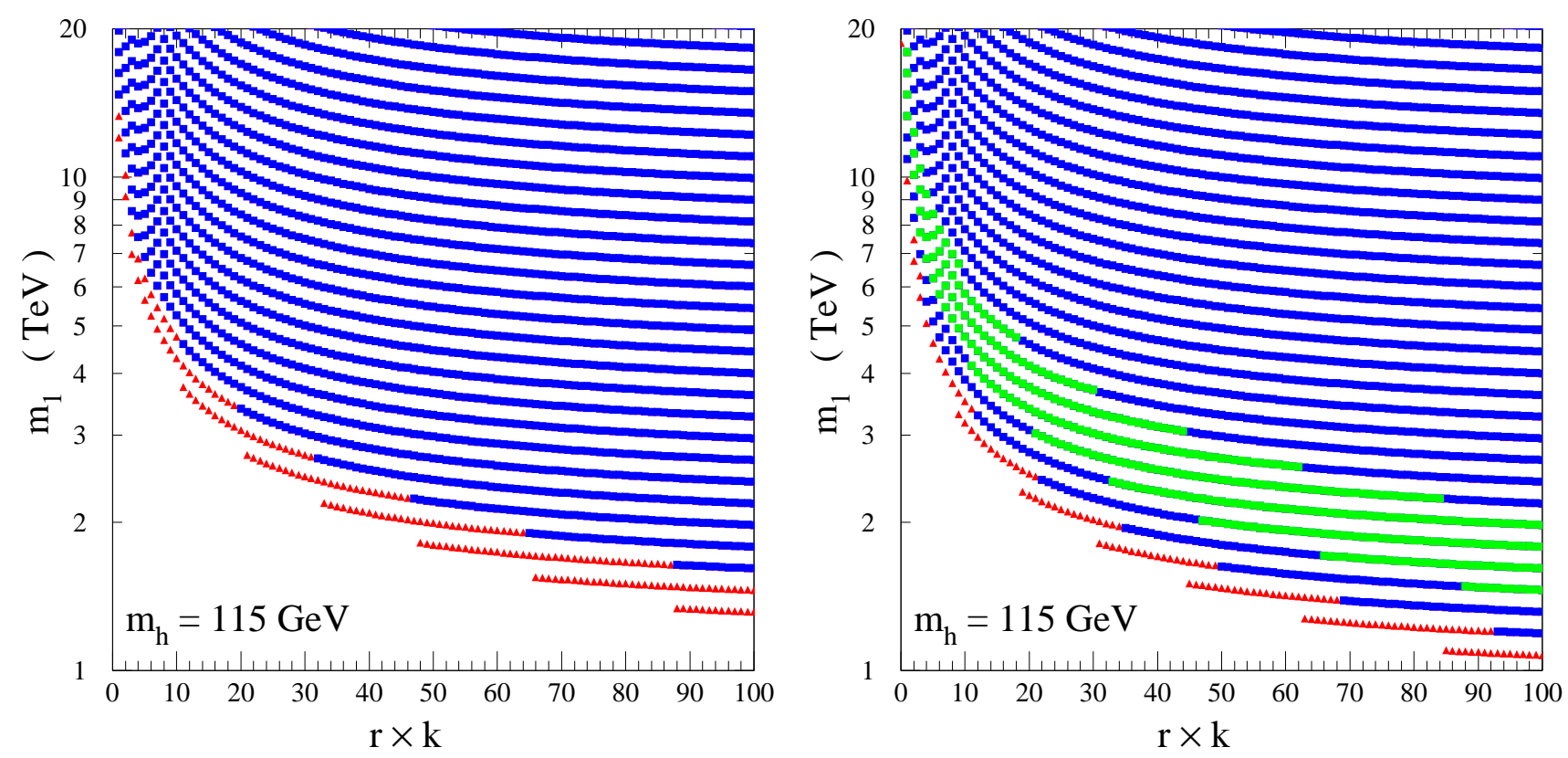

Figure 5: The regions of $1 \sigma$ [green (light grey) squares], $2 \sigma$ [blue (dark grey) squares] and $3 \sigma$ [red triangles] agreement with precision electroweak observables as defined in the text, in the plane of $r \times k$ and the mass of the first KK photon. The Higgs mass has been fixed to $m_{h}=115$ $\mathrm{GeV}$. The left figure includes all precision data in the fit, whereas the right figure includes only leptonic determinations of $\sin ^{2} \theta_{W}$.

by the RS model are within $n-\sigma$ error intervals of the central fitted values. We fix the top quark mass to its experimental mean value, $m_{t}=174.3 \mathrm{GeV}$. The one-sigma variations of the top quark mass lead to corrections of about $\Delta S_{t} \simeq \pm 0.01, \Delta T_{t} \simeq \pm 0.06$ and $\Delta U_{t} \simeq \pm 0.025$, which are smaller than the one $\sigma$ errors on $S, T$ and $U$ obtained from the fit to the data and do not affect our results in a relevant way.

When comparing how well RS fits the data compared to the SM, it is important to remember that the SM fails this analysis for any region of parameters at $1 \sigma$, and agrees at roughly $2 \sigma$ for Higgs masses above the LEP limit and below about $200 \mathrm{GeV}$. Furthermore, for the same range of Higgs mass parameters, removing $A_{F B}^{b}$ does not improve the agreement between the experimental data and the standard model predictions.

The results for $m_{h}=115 \mathrm{GeV}$ are shown in Fig. 5] plotted in the plane of $r$ and $m_{1}$, the mass of the first KK mode of the photon. Generally, the masses of the first KK modes of the $W$ and $Z$ bosons will be somewhat larger, because of the electroweak symmetry-breaking effects. 
In the limit $r \rightarrow 0$, we find agreement with the limit obtained in Ref. [4, $m_{1} \gtrsim 27 \mathrm{TeV}$. As $r$ increases, there are two different effects. For small non-zero $r$, the overall fit to the data remains roughly the same as the $r=0$ case (in other words, the properties of the zero modes do not change much), but the properties of the first KK mode and its coupling to fermions are quite radically affected, with both dropping. ${ }^{9}$ This results in the KK photon being much lighter than one would have assumed for zero $r$, and, for moderate values of $r$, its coupling is comparable to that of the zero mode photon.

For $r \times k \sim 20$ and $m_{1}$ on the order of $3.5 \mathrm{TeV}$, the fit to the entire data set is roughly as good as the SM itself, and the fit to the data without the hadron asymmetries is actually consistent within $1 \sigma$. Indeed, for the large region of parameters denoted by light squares in Fig. 5, we obtain an improvement of the fit to the data with respect to the Standard Model, with $m_{1}$ of order of a few TeV.

In Fig. 6] we show the same analysis, but with the Higgs mass fixed to $m_{h}=200 \mathrm{GeV}$. We see some small variation of the allowed regions, but by relatively small amounts, indicating that the RS fit does not prefer a light Higgs, but fits about equally well to Higgs masses as large as $200 \mathrm{GeV}$. The analysis for larger $m_{h}$ is straight-forward, and shows somewhat worse agreement than for $m_{h}=200 \mathrm{GeV}$.

Our results have very important implications for the RS model with gauge fields in the bulk. The preferred parameter space, for moderate $50 \gtrsim k r \gtrsim 10$, has a first KK photon with fermionic couplings of the order of the zero mode couplings, and a mass of a few TeV, most likely within reach of the LHC experiments.

\section{Conclusions}

The RS model is an interesting construction, with a novel solution to the hierarchy problem. While the simplest versions have gauge fields confined to the IR brane, there are interesting motivations to allow them to propagate into the extra dimension, including grand unification and a relation to a class of walking technicolor theories through the ADS/CFT correspondance. In that case, the lowest KK modes of the gauge bosons are usually very strongly coupled to fermions on the IR brane, with the lowest mass given by a factor of a few times $k e^{-k L}$, at the TeV scale.

\footnotetext{
${ }^{9}$ Note that, for $v / k \ll 1$, as demanded by the fit to the data, the properties of the KK mode masses and couplings can be read from Fig. 1.
} 

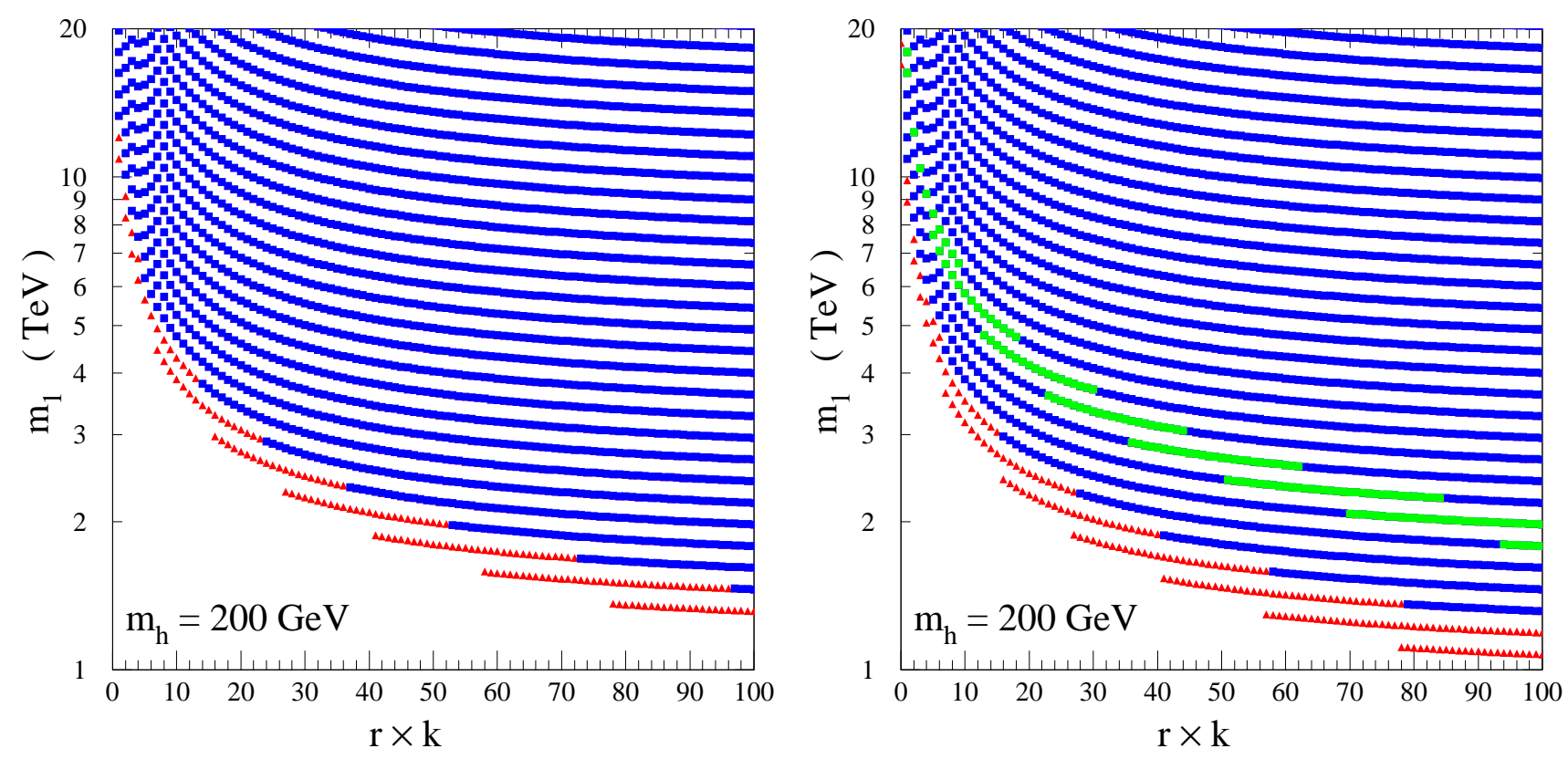

Figure 6: The same as Fig. 5 , but for $m_{h}=200 \mathrm{GeV}$.

Precision electroweak observables are challenging to fit in this framework, owing to corrections to the zero mode $W$ and $Z$ boson wave functions induced by the presence of the Higgs boson in the infra-red brane. In the absence of local gauge kinetic terms, consistency of the fit to the precision electroweak observables at the $2 \sigma$ level, as defined in the text, demands masses of the KK modes of the gauge bosons larger than about $27 \mathrm{TeV}$, beyond the reach of the next generation of collider experiments.

However, the inclusion of brane kinetic terms is helpful in lowering this bound. For moderately large values of $r, 2-\sigma$ consistency may be obtained for KK gauge boson masses of the order of a few TeV. Furthermore, if the hadronic asymmetries are excluded from the fit, the bulk gauge bosons may lead to a consistent fit to the data at the $1 \sigma$ level for a lightest KK mode of the order of a few $\mathrm{TeV}$ and Higgs masses in the range 115-200 GeV. These light KK modes have couplings of order of the zero mode coupling, opening new exciting possibilities at the LHC. 


\section{Acknowledgements}

The authors are grateful for conversations with G. Burdman. Work at ANL is supported in part by the US DOE, Div. of HEP, Contract W-31-109-ENG-38. Fermilab is operated by Universities Research Association Inc. under contract no. DE-AC02-76CH02000 with the DOE.

Note added: Related work on this subject appeared at a similar time in Ref. [27]. We have explored the case $r<0$ proposed in this reference and we found that the bounds from $S_{\text {eff }}, T_{\text {eff }}$, and $U_{\text {eff }}$ are more constraining than those based on the $\mathrm{KK}$ mode contributions to the muon decay constant alone.

\section{References}

[1] N. Arkani-Hamed, S. Dimopoulos and G. R. Dvali, Phys. Lett. B 429, 263 (1998) arXiv:hep-ph/9803315; I. Antoniadis, N. Arkani-Hamed, S. Dimopoulos and G. R. Dvali, Phys. Lett. B 436, 257 (1998) arXiv:hep-ph/9804398.

[2] L. Randall and R. Sundrum, Phys. Rev. Lett. 83, 3370 (1999) arXiv:hep-ph/9905221.

[3] H. Davoudiasl, J. L. Hewett and T. G. Rizzo, Phys. Lett. B 473, 43 (2000) arXiv:hep-ph/9911262.

[4] C. Csaki, J. Erlich and J. Terning, Phys. Rev. D 66, 064021 (2002) arXiv:hep-ph/0203034.

[5] G. Burdman, Phys. Rev. D 66, 076003 (2002) arXiv:hep-ph/0205329.

[6] S. J. Huber and Q. Shafi, Phys. Rev. D 63, 045010 (2001) arXiv:hep-ph/0005286; S. J. Huber, C. A. Lee and Q. Shafi, Phys. Lett. B 531, 112 (2002) arXiv:hep-ph/0111465.

[7] G. R. Dvali, G. Gabadadze and M. A. Shifman, Phys. Lett. B 497, 271 (2001) arXiv:hep-th/0010071.

[8] H. Georgi, A. K. Grant and G. Hailu, Phys. Lett. B 506, 207 (2001) arXiv:hep-ph/0012379. 
[9] M. Carena, T. M. P. Tait and C. E. M. Wagner, Acta Physica Polonica, B33, 2355 (2002) arXiv:hep-ph/0207056

[10] M. Carena, A. Delgado, J. Lykken, S. Pokorski, M. Quiros and C. E. Wagner, Nucl. Phys. B 609, 499 (2001) arXiv:hep-ph/0102172.

[11] G. R. Dvali, G. Gabadadze, M. Kolanovic and F. Nitti, Phys. Rev. D 64, 084004 (2001) arXiv:hep-ph/0102216.

[12] L. Randall and M. D. Schwartz, JHEP 0111, 003 (2001) arXiv:hep-th/0108114; Phys. Rev. Lett. 88, 081801 (2002) arXiv:hep-th/0108115; K. w. Choi, H. D. Kim and Y. W. Kim, arXiv:hep-ph/0202257; K. Agashe, A. Delgado and R. Sundrum, Nucl. Phys. B 643, 172 (2002) arXiv:hep-ph/0206099; A. Lewandowski, M. J. May and R. Sundrum, arXiv:hep-th/0209050, K. Agashe, A. Delgado and R. Sundrum, arXiv:hep-ph/0212028.

[13] W. D. Goldberger and I. Z. Rothstein, arXiv:hep-th/0204160 arXiv:hep-th/0208060

[14] E. Pontón and E. Poppitz, JHEP 0106, 019 (2001) arXiv:hep-ph/0105021.

[15] A. Pomarol, Phys. Lett. B 486, 153 (2000) arXiv:hep-ph/9911294.

[16] M. E. Peskin and T. Takeuchi, Phys. Rev. D 46, 381 (1992).

[17] T. G. Rizzo and J. D. Wells, Phys. Rev. D 61, 016007 (2000) arXiv:hep-ph/9906234.

[18] http://lepewwg.web.cern.ch/LEPEWWG/lepww/tgc/

[19] N. Arkani-Hamed, M. Porrati and L. Randall, JHEP 0108, 017 (2001) arXiv:hep-th/0012148.

[20] D. C. Kennedy and B. W. Lynn, Nucl. Phys. B 322, 1 (1989).

[21] G. Altarelli, F. Caravaglios, G. F. Giudice, P. Gambino and G. Ridolfi, JHEP 0106, 018 (2001) arXiv:hep-ph/0106029.

[22] C. Csaki, M. L. Graesser and G. D. Kribs, Phys. Rev. D 63, 065002 (2001) arXiv:hep-th/0008151.

[23] G. F. Giudice, R. Rattazzi and J. D. Wells, Nucl. Phys. B 595, 250 (2001) arXiv:hep-ph/0002178. 
[24] M. S. Chanowitz, Phys. Rev. Lett. 87, 231802 (2001) arXiv:hep-ph/0104024.

[25] D. Choudhury, T. M. P. Tait and C. E. M. Wagner, Phys. Rev. D 65, 053002 (2002) arXiv:hep-ph/0109097.

[26] P. Langacker, arXiv:hep-ph/0211065 LEP Electroweak Working Group, http://lephiggs.web.cern.ch/LEPEWWG/.

[27] H. Davoudiasl, J. L. Hewett and T. G. Rizzo, arXiv:hep-ph/0212279. 